The Astrophysical Journal SuPPLEMENT SeriEs, 138:75-98, 2002 January

(C) 2002. The American Astronomical Society. All rights reserved. Printed in U.S.A.

\title{
THE ORGANIC REFRACTORY MATERIAL IN THE DIFFUSE INTERSTELLAR MEDIUM: MID-INFRARED SPECTROSCOPIC CONSTRAINTS
}

\author{
Y. J. Pendleton ${ }^{1}$ AND L. J. Allamandola ${ }^{1}$ \\ Received 2001 March 22; accepted 2001 June 25
}

\begin{abstract}
This is an analysis of the $4000-1000 \mathrm{~cm}^{-1}(2.5-10 \mu \mathrm{m})$ region of the spectrum of diffuse interstellar medium (DISM) dust compared with the spectra of 13 materials produced in the laboratory which serve as analogs to the interstellar material. The organic signatures of extragalactic dust, carbonaceous chondritic material, and E. coli bacteria are also presented because these have been discussed in the literature as relevant to the diffuse interstellar medium. Spectral analysis of the DISM allows us to place significant constraints on the applicability of proposed candidate materials. The spectra of candidate materials are evaluated using four spectral characteristics based on the interstellar data: (i) comparisons of the profile and subpeak positions of the $2940 \mathrm{~cm}^{-1}(3.4 \mu \mathrm{m})$ aliphatic $\mathrm{CH}$ stretching-mode band, (ii) the ratio of the optical depth (O.D.) of the aliphatic CH stretch to the O.D. of the OH stretch near 3200 $\mathrm{cm}^{-1}(3.1 \mu \mathrm{m})$, (iii) the ratio of the O.D. of the aliphatic CH stretch to the O.D. of the carbonyl band near $1700 \mathrm{~cm}^{-1}(5.9 \mu \mathrm{m})$, and (iv) the ratio of the O.D. of the aliphatic $\mathrm{CH}$ stretch feature to the O.D. of the $\mathrm{CH}$ deformation modes near $1470 \mathrm{~cm}^{-1}(6.8 \mu \mathrm{m})$ and $1370 \mathrm{~cm}^{-1}(7.25 \mu \mathrm{m})$. We conclude that the organic refractory material in the diffuse interstellar medium is predominantly hydrocarbon in nature, possessing little nitrogen or oxygen, with the carbon distributed between the aromatic and aliphatic forms. Long alkane chains $\mathrm{H}_{3} \mathrm{C}-\left(\mathrm{CH}_{2}\right)_{\mathrm{n}}$ - with $n$ much greater than 4 or 5 are not major constituents of this material. Comparisons to laboratory analogs indicate the DISM organic material resembles plasma processed pure hydrocarbon residues much more so than energetically processed ice residues. This result is consistent with a birth site for the carrier of the $3.4 \mu \mathrm{m}$ band in the outflow region of evolved carbon stars. The organic material extracted from the Murchison carbonaceous meteorite and the spectrum of E. coli bacteria reveal spectral features in the 5-10 $\mu \mathrm{m}$ region that are absent in the DISM. Although the presence of unaltered circumstellar components in the Murchison meteorite has been established through several lines of evidence, it is unclear whether or not the aliphatic component which gives rise to the $3.4 \mu \mathrm{m}$ band is in that category. Considering the complete $2-10 \mu \mathrm{m}$ wavelength region, there is no spectral evidence for a biological origin of the $3.4 \mu \mathrm{m}$ interstellar absorption band. The similarity of the aliphatic $\mathrm{CH}$ stretch region of dust from our own Galaxy compared with that of distant galaxies suggests that the organic component of the ISM is widespread and may be an important universal reservoir of prebiotic organic carbon.
\end{abstract}

Subject headings: infrared: ISM: lines and bands - ISM: molecules - molecular data

\section{INTRODUCTION}

During the past 25 years, our understanding of the solidstate component of the interstellar medium has grown from supposition to firm chemical classifications due to the tremendous strides made in infrared astronomical observations and advances in laboratory studies of materials produced under simulated interstellar conditions. One of the most intriguing tracers of the solid state organic component of the diffuse interstellar medium (DISM) has been the $2940 \mathrm{~cm}^{-1}(3.4 \mu \mathrm{m})$ absorption band. Since its detection along the line of sight toward infrared source number 7 in the Galactic center (Sgr A W, IRS 7) by Willner et al. (1979) and Wickramasinghe \& Allen (1980), follow-up studies have shown that this material is likely a common DISM component in the Milky Way and other galaxies (Butchart et al. 1986; Adamson, Whittet, \& Duley 1990; Sandford et al. 1991; Pendleton et al. 1994; Wright et al. 1996; Pendleton 1996a, 1996b, 1997; Whittet et al. 1997; Imanishi \& Dudley 2000; Imanishi 2000). Although the $3.4 \mu \mathrm{m}$ spectral band probes only the $\mathrm{CH}$ stretching modes of the carrier, band position and profile analysis have revealed that this portion

\footnotetext{
${ }^{1}$ NASA Ames Research Center, Mail Stop 245-3, Moffett Field, CA 94035 .
}

of the refractory material contains aliphatic hydrocarbons (open chain $-\mathrm{CH}_{2}-$, and $-\mathrm{CH}_{3}$ groups) that incorporate at least $3 \%-4 \%$ of the available interstellar carbon. Further, the band profile shows that these aliphatic groups are attached to larger chemical structures which likely contain more than $10 \%$ of the total available interstellar carbon (Sandford et al. 1991; Pendleton et al. 1994).

Over the past 20 years, nearly two dozen different materials have been proposed as analogs of the interstellar organic refractory material, based on reasonably good matches to the $2940 \mathrm{~cm}^{-1}(3.4 \mu \mathrm{m})$ spectral feature. Some were produced under conditions which mimic interstellar conditions, designed to yield insight into possible interstellar production sites, while others sought only to reproduce details of the spectrum, in order to identify the material. Here we assess a representative sample of 13 lab residue materials which have been suggested as possible analogs of the solid-state organic component in the DISM based on comparisons to the $3.4 \mu \mathrm{m}$ band. By considering the complete mid-IR spectrum from 4000 to $1000 \mathrm{~cm}^{-1}(2.5$ to $10 \mu \mathrm{m}$ ) of each candidate material, and by comparing it to data available for the DISM, we narrow the list of possible candidates and, in so doing, hope to clarify the direction further experimental and observational studies need to take in the quest to characterize the organic component of 

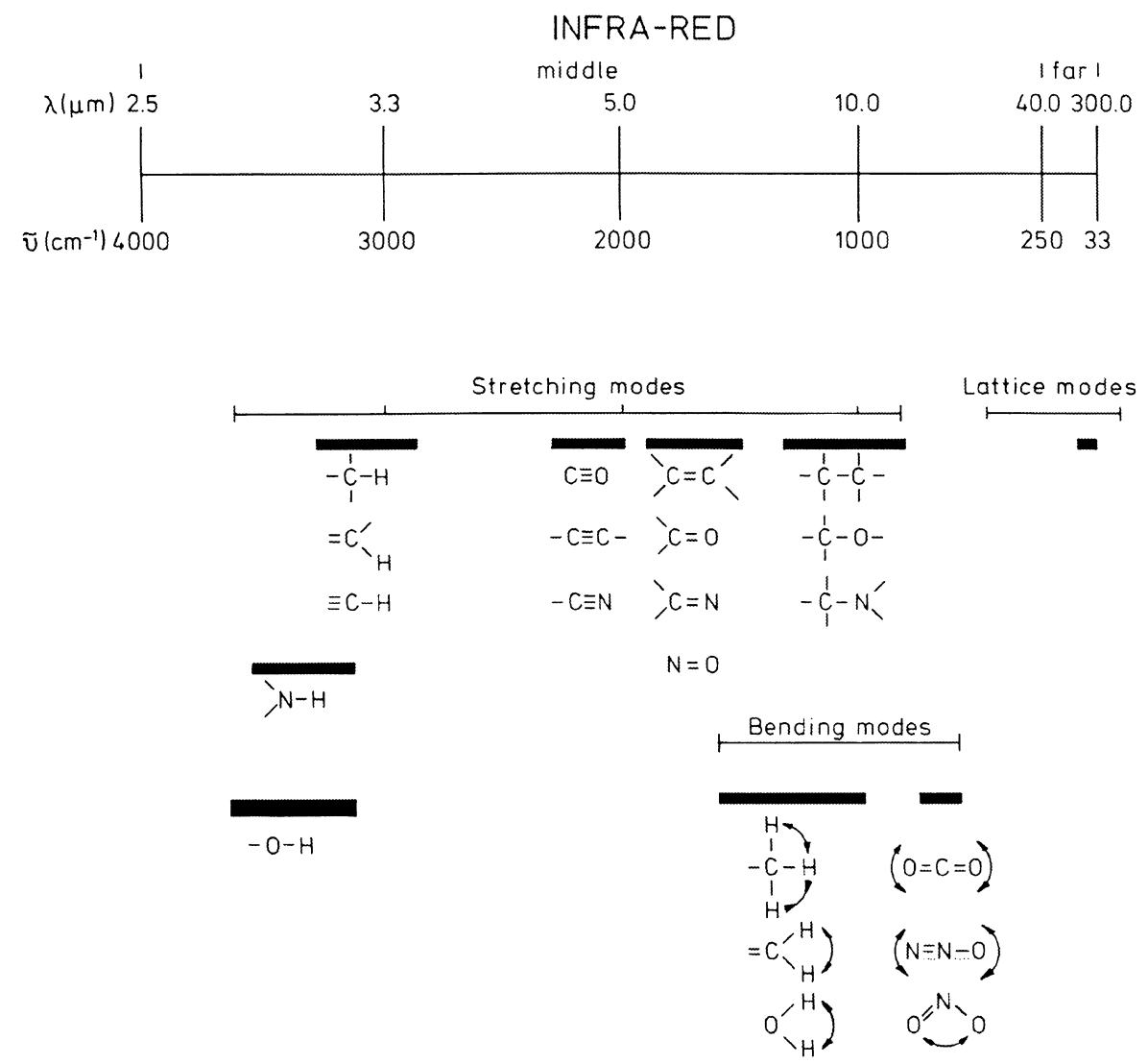

Fig. 1. - Characteristic vibrational frequencies for chemical subgroups in the 4000-400 $\mathrm{cm}^{-1}(2.5-25 \mu \mathrm{m})$ region of the infrared (Reproduced from Allamandola 1984).

the diffuse interstellar medium. All of the materials presented here "fit" the infrared interstellar data near $2940 \mathrm{~cm}^{-1}$ $(3.4 \mu \mathrm{m})$ to lesser or greater degrees. However, the noteworthy absence of any "strong" spectral features in the interstellar data in the $\mathrm{OH}$ and $\mathrm{NH}$ stretching region (3500$\left.3000 \mathrm{~cm}^{-1}, 2.86-3.3 \mu \mathrm{m}\right)$ and at the lower frequencies, $2000-1250 \mathrm{~cm}^{-1}(5-8 \mu \mathrm{m})$, severely constrains the overall chemical nature of the material responsible for the interstellar absorption centered near $2940 \mathrm{~cm}^{-1}(3.4 \mu \mathrm{m})$, hereafter referred to as the $3.4 \mu \mathrm{m}$ band (as is customary in the astrophysical literature).

We evaluate the spectra of candidate materials using four spectral characteristics: (i) comparisons of the $2940 \mathrm{~cm}^{-1}$ $(3.4 \mu \mathrm{m})$ aliphatic $\mathrm{CH}$ stretch band profile and subpeak positions, (ii) the ratio of the optical depth $(\tau)$ of the aliphatic $\mathrm{CH}$ stretch to the $\tau$ of the $\mathrm{OH}$ stretch near 3200 $\mathrm{cm}^{-1}(3.1 \mu \mathrm{m})$, (iii) the ratio of the $\tau$ of the aliphatic $\mathrm{CH}$ stretch to the $\tau$ of the carbonyl band near $1700 \mathrm{~cm}^{-1}(5.9$ $\mu \mathrm{m}$ ), and (iv) the ratio of the $\tau$ of the aliphatic $\mathrm{CH}$ stretch feature to the $\tau$ of the $\mathrm{CH}$ deformation modes near 1470 $\mathrm{cm}^{-1}(6.8 \mu \mathrm{m})$ and $1370 \mathrm{~cm}^{-1}(7.25 \mu \mathrm{m})$.

Striking similarities between the $3.4 \mu \mathrm{m}$ interstellar band and the corresponding aliphatic $\mathrm{CH}$ stretch in the organic extracts of the Murchison and Orgueil carbonaceous meteorites suggest that some of the interstellar material originally incorporated into the solar nebula may have survived relatively unaltered in primitive solar system bodies (Ehrenfreund et al. 1991; Pendleton et al. 1994). The enhanced deuterium abundance in the organic component and other isotopic anomalies, ensures that part of the extracted material is extraterrestrial (Kerridge, Chang, \&
Shipp 1987; Ming \& Anders 1988; Lewis, Amari, \& Anders 1994), and the presence of isotopically anomalous diamonds, silicon carbide ( $\mathrm{SiC})$, titanium carbide (TiC), and graphite confirms that interstellar material survived subsequent processing on the meteorite parent bodies (cf. Anders \& Zinner 1993). More detailed accounts of the composition of the Murchison meteorite can be found in Cronin, Pizzarello, \& Cruikshank (1988), Bernatowicz et al. (1996), and Cronin \& Pizzarello (1990). Here, in addition to laboratory analogs, we also compare the $2-10 \mu \mathrm{m}$ spectrum of the sublimate from the acid and organic insoluble kerogen from the Murchison meteorite (DeVries et al. 1993; Kerridge et al. 1987) to the interstellar data. A brief discussion of the relevance of the interstellar component in meteorites to the evolution of dust in the ISM is presented.

The literature also contains comparisons of the interstellar near-infrared spectral features (between 3 and $4 \mu \mathrm{m}$ ) to those of the E. coli bacteria, first suggested in the early discovery period before high resolution interstellar spectra were available. Here we present the entire $2-10 \mu \mathrm{m}$ spectrum of E. coli to allow a complete comparison of the interstellar spectrum with that of the bacterium, and we conclude the interstellar $3.4 \mu \mathrm{m}$ band does not have a biological origin.

The DISM $3.4 \mu \mathrm{m}$ band in our own Galaxy closely matches recent detections in other galaxies. Here we present a comparison between the luminous galaxy IRAS $08572+3915$ to the DISM spectrum. Given that the carrier of the $3.4 \mu \mathrm{m}$ interstellar absorption band appears to be widespread throughout our Galaxy and is present in several others (Pendleton 1996a, 1996b, 1997; Wright et al. 1996; 


\section{H-X STRETCHING FREQUENCIES}

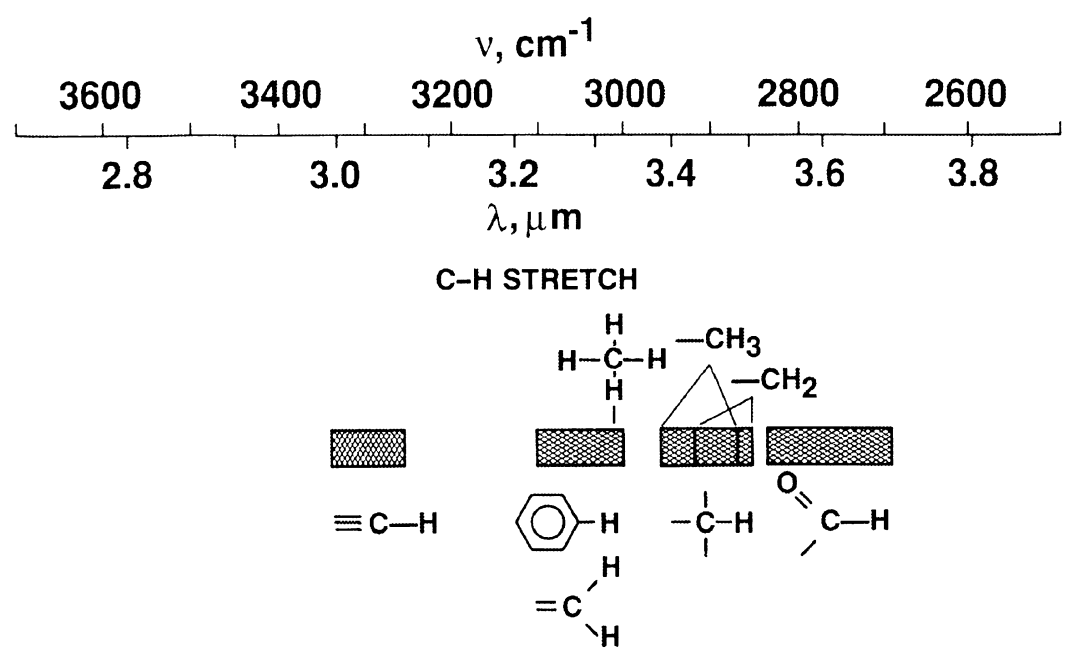

N-H STRETCH

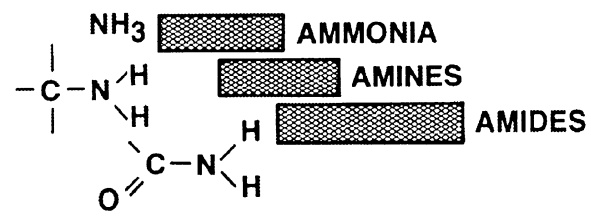

O-H STRETCH

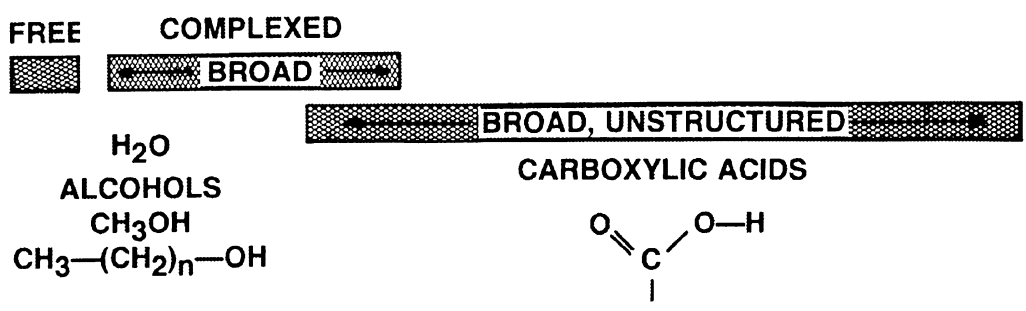

Fig. 2.-Breakdown of the characteristic vibrational frequencies in the $\mathrm{NH}, \mathrm{OH}$, and $\mathrm{CH}$ stretch region according to $\mathrm{NH}$, $\mathrm{OH}$, and $\mathrm{CH}$ type. The unlabeled $\mathrm{CH}$ stretching domains (from higher to lower frequencies) correspond to acetylenic, aromatic and olefinic, aliphatic, and aldehydic $\mathrm{CH}$ stretches.

Imanishi \& Dudley 2000), it may be an important universal reservoir of prebiotic organic carbon.

This paper is organized as follows. Salient principles of infrared spectroscopy applicable to this analysis are summarized in $\S 2$. Section 3 presents the mid-infrared spectrum of the DISM as represented by the line of sight toward Cygnus OB2 No. 12, an infrared bright background source through which intervening diffuse interstellar medium dust is observed. Spectra taken from the NASA Infrared Telescope Facility (IRTF), the Kuiper Airborne Observatory (KAO), and the Infrared Space Observatory (ISO) are compared and the constraints these spectra impose on the laboratory candidate materials are quantified. The infrared spectra of the various candidate materials are discussed in $\S 4$, followed by a comparison of the complete $4000-1000$ $\mathrm{cm}^{-1}(2.5-10 \mu \mathrm{m})$ spectrum of each sample to the interstellar spectrum. In $\S 5$, the laboratory spectra are evaluated based on the spectral constraints of the DISM dust. The overall characteristics of the DISM organic refractory material as indicated by the spectrum toward Cygnus OB2 No. 12 and astrophysical implications based on the evaluations presented are given in $\S 6$. Conclusions of the paper are summarized in $\S 7$.

\section{MID-INFRARED MOLECULAR SPECTROSCOPY}

Within a molecule, chemical subgroups of atoms vibrate at characteristic frequencies for that group, giving rise to infrared absorption or emission bands that always fall at or near the same position in the spectrum, regardless of the rest of the molecule or material present. For this reason, chemists have long used the infrared spectrum of any material as a diagnostic of the chemical subgroups present (e.g., Bellamy 1960; Silverstein \& Bassler 1967). The chemistry of the ISM is dominated by the cosmically abundant $\mathrm{H}, \mathrm{C}, \mathrm{O}$, and $\mathrm{N}$ atoms, and diagnostic bands from combinations of these atoms fall almost exclusively in the nearand mid-infrared region between 4000 and $400 \mathrm{~cm}^{-1}(2.5$ and $25 \mu \mathrm{m}$, e.g., Allamandola 1984). Thus, infrared spectra are routinely used to classify interstellar species and subgroups by chemical type (i.e., aliphatic vs. aromatic hydrocarbons vs. carbon chains; carbonyl vs. alcoholic carbon-oxygen links, etc.) and, in the case of mixed materials, to characterize the contributing types. For example, Sloan et al. (1997) and Bernstein et al. (1996) describe interstellar polycyclic materials which contain both aliphatic and aromatic $\mathrm{CH}$. Figure 1 provides a guide 
to the diagnostic spectral bands of mid-infrared vibrations from chemical subgroups comprised of $\mathrm{H}$ and the most cosmically abundant, reactive heavy elements $(\mathrm{O}, \mathrm{C}$, and $\mathrm{N})$. Figure 2 provides a detailed look at those bands which are active in the 3.2-3.6 $\mu \mathrm{m}$ region. From these two figures it is clear that any hydrocarbon will exhibit activity in the $3.0-3.4 \mu \mathrm{m}$ region, and there will be corresponding activity in the 5-8 $\mu \mathrm{m}$ region, depending on the exact composition and configuration of the larger molecules containing the spectrally active subgroups.

\section{THE $4000-1000 \mathrm{~cm}^{-1}(2-10 \mu \mathrm{m})$ SPECTRUM OF Cyg OB2 No. 12 AND THE DIFFUSE INTERSTELLAR MEDIUM}

Classified as one of the most luminous stars in the Galaxy, Cyg OB2 No. 12 has a spectral type of $\mathrm{B} 5 \mathrm{Ia}^{+}$, absolute magnitude, $M_{v}=-9.9$, and a visual extinction of $A_{v}=10.2$ (Humphreys 1978). Its distance is $1.7 \pm 0.2 \mathrm{kpc}$ (Torres-Dodgen, Carroll, \& Tapia 1991), but it suffers a greater amount of extinction than would be expected purely based on its distance. The intervening dust has been well studied (Rieke 1974; Gillett, Forrest, \& Merrill 1973; Persi \& Ferrari-Toniolo 1982; Roche \& Aitken 1984; Adamson et al. 1990; Sandford et al. 1991; Pendleton et al. 1994; Whittet et al. 1997, 2001, among others), and the common conclusion is that the extinction along the sight line toward Cyg OB2 No. 12 samples only DISM dust with little or no contribution from dense, molecular cloud dust and ice. The enhanced quantity of dust is likely due to an uneven spatial distribution of intracluster material within the Cyg OB2 association (see McMillan \& Tapia 1977), and that dust exhibits properties typical of the DISM, such as the ratio of total-to-selective extinction $\left(R_{V}=A_{V} / E_{B-V}=2.90 \pm 0.15\right.$; Adamson et al. 1990). Silicate absorption near $10 \mu \mathrm{m}$ (Rieke 1974 ) is similar in profile to that seen in circumstellar emission around stars such as $\mu$ Cephei, suggesting structural and compositional similarity between the DISM dust and stardust ejected by evolved stars (Roche \& Aitken 1984, 1985; Bowey, Adamson, \& Whittet 1998).

Figure $3 a$ compares the $4000-1100 \mathrm{~cm}^{-1}(2.5-9 \mu \mathrm{m})$ spectrum of Cyg OB2 No. 12 taken from the Infrared Space Observatory using the Short Wavelength Spectrometer (SWS) (Whittet et al. 1997; solid line), the NASA IRTF using the Cooled Grating Array Spectrometer (CGAS) (Sandford et al. 1991; Pendleton et al. 1994; dashed line), and the Kuiper Airborne Observatory (KAO) using the High-resolution Infrared Observation Grating Spectrometer (HIFOGS) (Pendleton 1995; longer dashed line). The three data sets demonstrate good overall agreement in flux level, and confirmation that there are very few significant $(\tau>0.04)$ absorption features present other than those near $3.4 \mu \mathrm{m}$.

Figure $3 b$ compares the $3125-2630 \mathrm{~cm}^{-1}(3.2-3.8 \mu \mathrm{m})$ portion of the spectrum and Figure $3 c$ the $1800-1175 \mathrm{~cm}^{-1}$ $(5.5-8.5 \mu \mathrm{m})$ portion of the spectrum, taken with different spectrometers as outlined in Figure $3 a$. The very weak, broad absorption seen in Figure $3 b$ centered near $3.4 \mu \mathrm{m}$ has been identified as characteristic of material in the DISM through a program of study in which several sight lines through the DISM were compared (Sandford et al. 1991, Pendleton et al. 1994, and Whittet 1997). The profiles and band positions agree in cases where various types of stars are seen through intervening dust along different directions from the Earth. This has led to the conclusion that the band carrier resides in the DISM as it is highly unlikely that the features would be associated with the various types of stars. The weak absorptions present near $3.4 \mu \mathrm{m}$ have been studied in detail, and spectral comparisons with the line of sight toward the Galactic center, which has greater extinction, reveal the presence of aliphatic hydrocarbons (due to $-\mathrm{CH}_{2}-$ and $-\mathrm{CH}_{3}$ groups).

Studies of dust along the line of sight toward the Galactic center Sgr A* (Adamson et al. 1990; Sandford et al. 1991; Pendleton et al. 1994; Sandford, Pendleton, \& Allamandola 1995) have shown that this sight line contains a contribution from both diffuse and dense cloud dust (Tielens et al. 1996; Gerakines et al. 1999; de Graauw et al. 1996; Lutz et al. 1996). However, the $3125-2630 \mathrm{~cm}^{-1}(3.2-3.8 \mu \mathrm{m})$ region is relatively unaffected by the presence of the dense cloud material (Tielens et al. 1996; Chiar et al. 2000). Furthermore, Figure 4 demonstrates that when the optical depths are scaled for comparison, the spectral signature in the 3.4 $\mu \mathrm{m}$ region of the dust seen toward CYG OB2 No. 12 is indeed similar to the dust seen toward the Galactic center (Sandford et al. 1991; Pendleton et al. 1994). The interstellar extinction toward the Galactic center is a factor of 3 higher than that toward Cyg OB2 No. 12, and the higher column density of dust provides more detailed profile information. Due to the brightness of the Galactic center in the infrared, higher signal-to-noise ratio data have been obtained in studies of dust along that line of sight. For both of these reasons, we have elected to use the Galactic center spectrum of GC IRS 6E (Pendleton et al. 1994), for the profile comparisons with the spectra of candidate laboratory materials near $3.4 \mu \mathrm{m}$, which are shown with the full spectrum of the candidate materials in $\S 4$.

However, since the line of sight toward the Galactic center also samples dense cloud dust and ice, the features at longer wavelengths are severely blended with ice absorptions (Tielens et al. 1996; Chiar et al. 2000). Therefore, the longer wavelength constraints we place upon the DISM

TABLE 1

Tau (Optical Depth) Values and UpPer Limits Derived from the ISO Spectrum of Cyg OB2 No. 12 at the Frequencies Characteristic of the Fundamental

Stretching Vibrations of Hydroxy $(\mathrm{OH})$, Aliphatic $(\mathrm{CH})$, and Carbonyl $(\mathrm{C}=\mathrm{O})$ Groups, aNd the Deformation Vibration FreQuency of Aliphatic (CH)

\begin{tabular}{ccccc}
\hline \hline & \multicolumn{4}{c}{ Vibration } \\
\cline { 2 - 5 } Property & OH Stretch & CH Stretch & CO Stretch & CH Deform. \\
\hline Frequency peak $\ldots \ldots \ldots \ldots$ & $3300 \mathrm{~cm}^{-1}$ & $2490 \mathrm{~cm}^{-1}$ & $1700 \mathrm{~cm}^{-1}$ & $1450 \mathrm{~cm}^{-1}$ \\
Wavelength position $\ldots \ldots . .$. & $3 \mu \mathrm{m}$ & $3.4 \mu \mathrm{m}$ & $5.88 \mu \mathrm{m}$ & $6.9 \mu \mathrm{m}$ \\
Optical depth $(\tau) \ldots \ldots \ldots$. & $<0.02$ & 0.038 & $<0.06$ & $<0.04$ \\
\hline
\end{tabular}




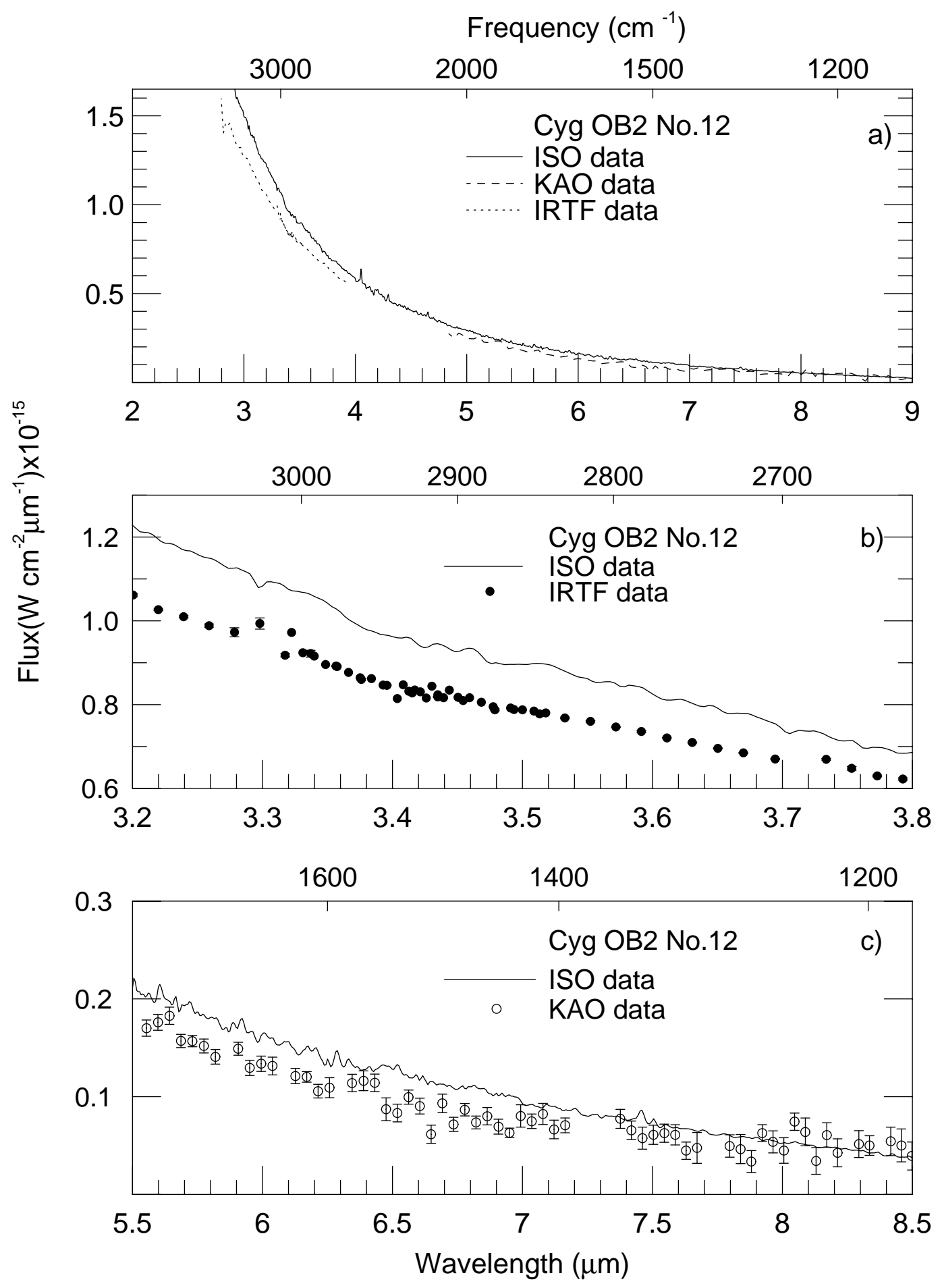

FIG. 3.-Comparison of the 2.5-9 $\mu \mathrm{m}$ spectrum of Cyg OB2 No. 12 taken from the NASA IRTF using the Cooled Grating Array Spectrometer (CGAS) (Sandford et al. 1991; Pendleton et al. 1994), the Kuiper Airborne Observatory (KAO) using the High Resolution Infrared Faint Object Grating Spectrometer (HIFOGS) (Pendleton 1995; Pendleton et al. 1999), and the Infrared Space Observatory (ISO) using the Short Wavelength Spectrometer (SWS) (Whittet et al. 1997). Panels show (a) the 3570-1100 $\mathrm{cm}^{-1}(2.5-9 \mu \mathrm{m})$ region, $(b)$ the $3125-2630 \mathrm{~cm}^{-1}(3.2-3.8 \mu \mathrm{m})$ region, and $(c)$ the $1800-1175 \mathrm{~cm}^{-1}(5.5-8.5$ $\mu \mathrm{m})$ region.

dust are based solely on the spectrum of Cyg OB2 No. 12 . Recent studies of the Galactic center by Chiar et al. (2000), in which an analysis is presented of the diffuse and dense components to decouple the two, are consistent with our results for the diffuse dust component of the ISM.

The DISM C-H stretching features fall at 2955, 2925, and $2870 \mathrm{~cm}^{-1}(3.38,3.42$, and $3.48 \mu \mathrm{m})$ and closely match the symmetric and asymmetric $\mathrm{C}-\mathrm{H}$ stretching frequencies of $-\mathrm{CH}_{3}$ (methyl) and $-\mathrm{CH}_{2}-$ (methylene) groups in saturated aliphatic hydrocarbons. The average $-\mathrm{CH}_{2}-/-\mathrm{CH}_{3}-$ ratio in this material is $2.5 \pm 0.4$, which suggests the hydrocarbon component of the DISM grain material contains structures like $-\mathrm{CH}_{2}-\mathrm{CH}_{2}-\mathrm{CH}_{3}$ and $-\mathrm{CH}_{2}-\mathrm{CH}_{2}-\mathrm{CH}_{2}-\mathrm{CH}_{3}$. As discussed in Sandford et al. 


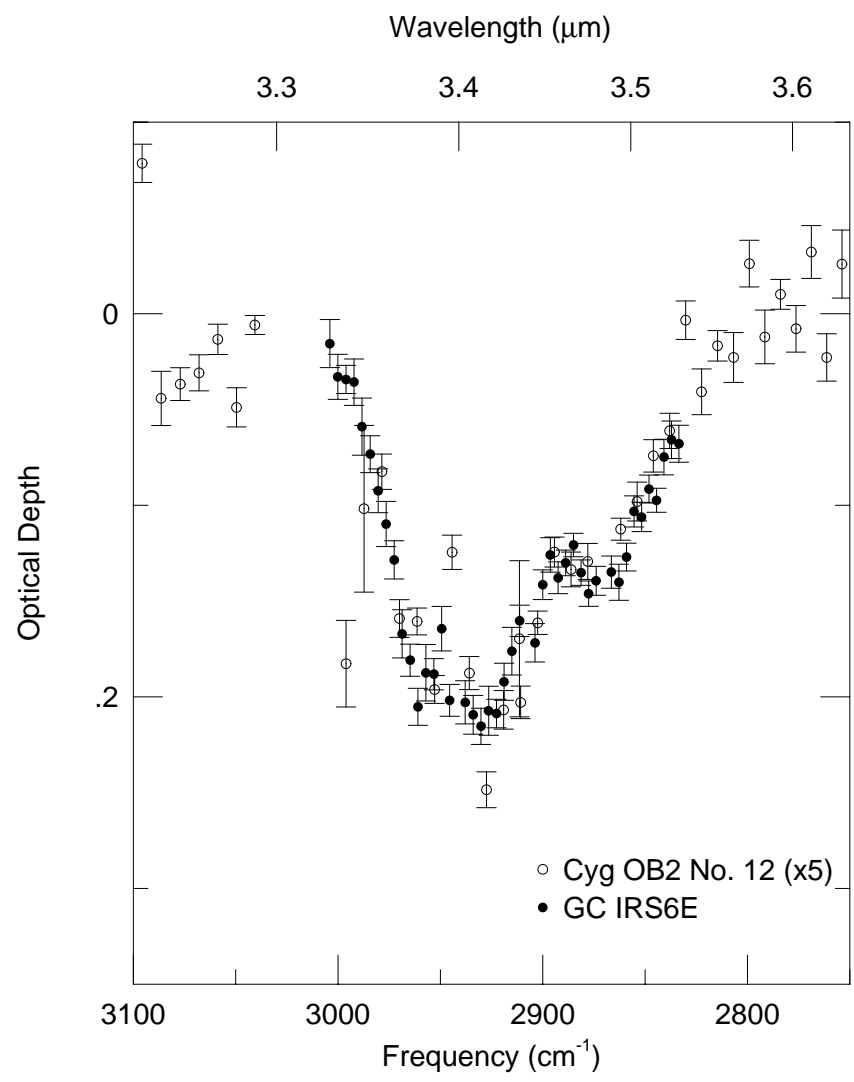

FIG. 4.-Comparison of the $\mathrm{CH}$ stretch feature toward Cyg OB2 No. 12 with that toward Galactic center source IRS 6E. Spectra are reproduced from Pendleton (1995) and Pendleton et al. (1994), respectively. The data for Cyg OB2 No. 12 have been multiplied by a factor of 5 to match the optical depth of the Galactic center data.

(1991), chemically bonded electronegative groups are close (but not adjacent) to the $-\mathrm{CH}_{2}-$ and $-\mathrm{CH}_{3}$ groups. The presence of any electronegative group perturbs the local fields within the molecule and affects the bond force constants, causing band shifts and broadening. The effect of the electronegative group diminishes with distance and only the nearer aliphatic groups are greatly altered. Thus, $-\mathrm{CH}_{2}-$ and $-\mathrm{CH}_{3}$ groups that are separated from the electronegative group by several intermediate carbon atoms will produce essentially aliphatic spectral features. The refractory carbonaceous component of DISM dust likely contains aromatic and diamond-like material interlinked by chains of saturated aliphatic hydrocarbons. These $\mathrm{C}-\mathrm{H}$ bands yield information only about the carbon atoms to which hydrogen is attached, and studies of the $3.4 \mu \mathrm{m}$ interstellar band alone cannot yield information about the bonding between the heavier elements such as carbon, oxygen, and nitrogen that make up the rest of the organic component. Figure 1 shows that vibrations involving heavier atoms appear between 5 and $10 \mu \mathrm{m}$. Therefore, estimates of the percent of cosmic carbon locked up in the 3.4 $\mu \mathrm{m} \mathrm{C}-\mathrm{H}$ feature (at least $2.5 \%$; Sandford et al. 1991; Pendleton et al. 1994) are lower limits to the fraction of carbon involved in the entire interstellar hydrocarbon complex.

As shown in Figure $3 c$, there is a general absence of prominent absorption features in the $1800-1100 \mathrm{~cm}^{-1}(5-9$ $\mu \mathrm{m})$ region of the Cyg OB2 No. 12 spectrum. However, the upper limits that can be placed on possible weak features are an important means of further investigating the dust composition in the DISM. The diffuse interstellar medium spectrum longward of $5 \mu \mathrm{m}$ as seen toward Cyg OB2 No. 12, first measured from the Kuiper Airborne Observatory in 1993 (e.g., see Figs. $3 a$ and 3c), was rather surprising because the 5-8 $\mu \mathrm{m}$ region appeared relatively featureless. There were weak hints of some type of organic material (Pendleton 1995), but the uncertainty in the data necessitated further testing. The absence of significant spectral structure at longer wavelengths has now been pushed to lower limits by the Infrared Space Observatory (Whittet \& Tielens 1997). Unfortunately, even with the ISO data, the signal precision remains insufficient to make firm detections of the weak features expected in the 5.5-8.5 $\mu \mathrm{m}$ range (see Fig. 3c). Given the solid detection of the $3.4 \mu \mathrm{m}$ band from ground-based observations and from ISO (Figs. $3 a, 3 b$, and 4), however, there are strong implications regarding the positions of additional features at different wavelengths. Optical depth upper limits at these positions are listed in Table 1.

\section{THE $4000-1000 \mathrm{~cm}^{-1}(2-10 \mu \mathrm{m})$ SPECTRA OF CANDIDATE MATERIALS}

A brief summary of the candidate materials considered will be presented in this section. Salient aspects of preparation are given for each substance, followed by a short discussion of the chemical composition indicated by the most striking features in the infrared spectrum of the material. The initial spectral discussions are more detailed than are the latter to illustrate the approach and to minimize repetition. When the spectroscopic case for a new subgroup for a given material is presented, more discussion is provided. All frequency assignments, as well as relative and intrinsic band strengths used, can be found in Bellamy (1960), Silverstein \& Bassler (1967), Wexler (1967), and d'Hendecourt \& Allamandola (1986). Band frequency regions are presented schematically in Figures 1 and 2. For the laboratory samples, the intent is simply to summarize what is indicated by the spectra without interpretation based on what might have occurred in the processing.

\subsection{Residues Produced in Ices Irradiated by Ultraviolet Light \\ 4.1.1. Residue from $\mathrm{H}_{2} \mathrm{O}: \mathrm{CH}_{3} \mathrm{OH}: \mathrm{CO}: \mathrm{NH}_{3}$ Irradiated Ice 4.1.1.1. Material Preparation}

This organic residue is produced by the in situ, vacuum ultraviolet (UV) photolysis of the mixed molecular ice $\mathrm{H}_{2} \mathrm{O}: \mathrm{CH}_{3} \mathrm{OH}: \mathrm{CO}: \mathrm{NH}_{3}$ (100:50:10:10) after deposition onto a substrate at $10 \mathrm{~K}$. The UV radiation consists of an intense, narrow line at $\operatorname{Ly} \alpha(1216 \AA)$ and a several hundred Angstroms wide band peaking near $1600 \AA$. The spectrum is of the residual material which remains on the substrate after the irradiated ice has been warmed up to room temperature under vacuum. The residue was then cooled to $10 \mathrm{~K}$ and the spectrum taken. This spectrum has been reported in Allamandola, Sandford, \& Valero (1988) and Bernstein et al. (1995).

\subsubsection{Infrared Properties}

The infrared spectrum of this material is shown in Figures $5 a$ and $6 a$. Unfortunately, the detector used during these experiments failed at frequencies higher than about $3000 \mathrm{~cm}^{-1}(3.33 \mu \mathrm{m})$. Nonetheless, the sloping baseline which starts near $2400 \mathrm{~cm}^{-1}(4.17 \mu \mathrm{m})$ is suggestive of a moderately strong, broad absorption underlying the nar- 


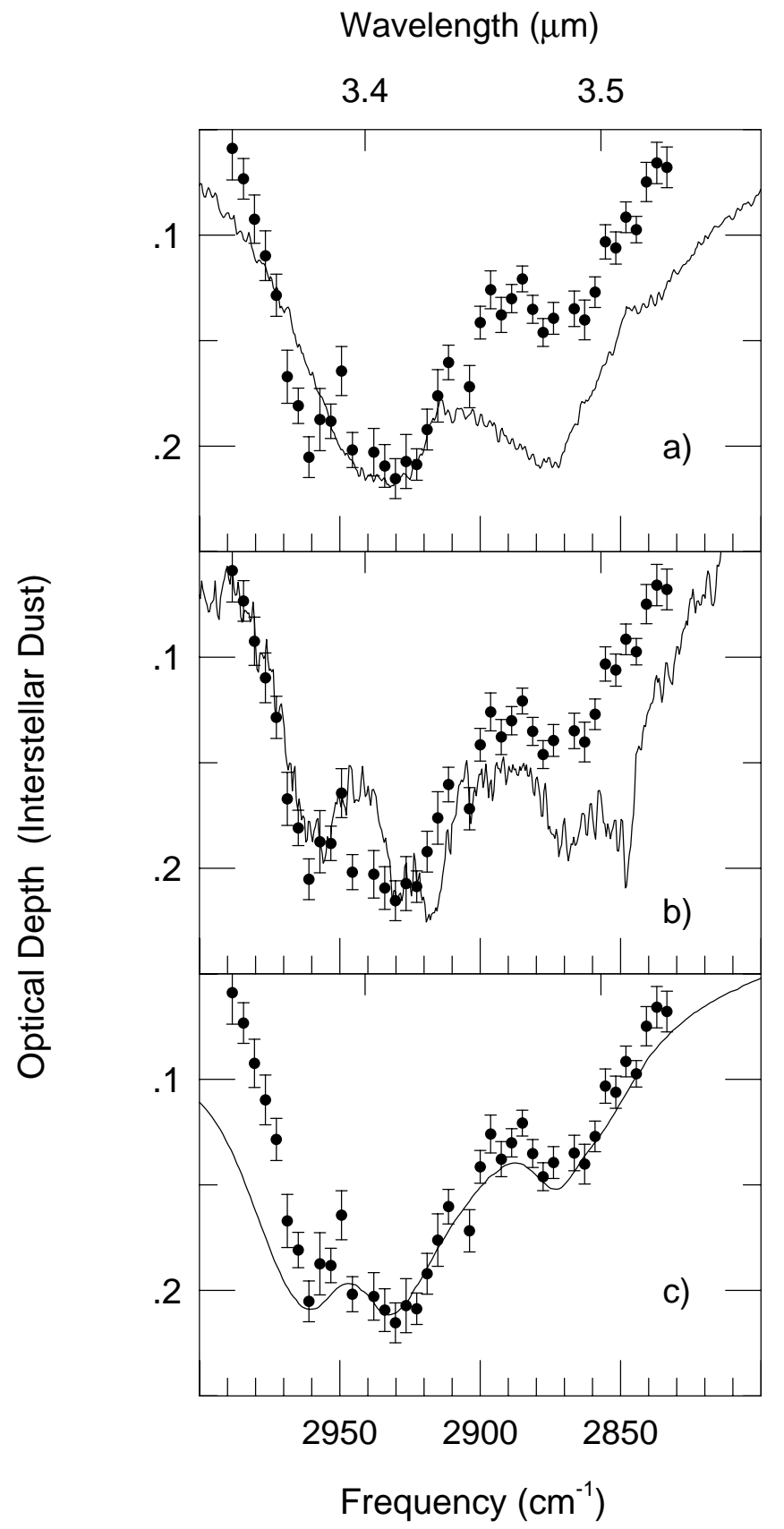

FIG. 5.-Comparison of the diffuse interstellar medium $3.4 \mu \mathrm{m}$ absorption features (points) to the corresponding band in the spectrum of the residue materials (lines) produced by the UV irradiation of various interstellar ice analogs as described in $\S 4.1$. The Diffuse Interstellar Medium spectrum (GC IRS 6E) is taken from Pendleton et al. (1994). Laboratory spectra: (a) $\mathrm{H}_{2} \mathrm{O}: \mathrm{CH}_{3} \mathrm{OH}: \mathrm{NH}_{3}: \mathrm{CO}(100: 50: 10: 10)$ Allamandola et al. (1988), Bernstein et al. (1995); (b) $\mathrm{H}_{2} \mathrm{O}: \mathrm{CH}_{3} \mathrm{OH}: \mathrm{NH}_{3}: \mathrm{CO}: \mathrm{C}_{3} \mathrm{H}_{8}$ (100:50:10:10:10) Allamandola et al. (1988); (c) $\mathrm{H}_{2} \mathrm{O}: \mathrm{CO}: \mathrm{NH}_{3}: \mathrm{C}_{2} \mathrm{H}_{6}$ (5:2:2:1) Greenberg et al. (1995).

rower doublet centered near $2900 \mathrm{~cm}^{-1}(3.45 \mu \mathrm{m})$. This may indicate an extensive hydrogen bonded network of $\mathrm{OH}$ and NH bonds (see Fig. 2 and Allamandola 1984). The extreme breadth of the feature and low frequency limit indicates the presence of an acidic group. Given the organic nature of this material, this feature probably arises from carboxylic acid groups $(-\mathrm{COOH})$. Other compounds which contain $\mathrm{OH}$, such as water and alcohols, are likely present as well, with their strong, broad, $\mathrm{H}$-bonded $\mathrm{OH}$ stretch band blending

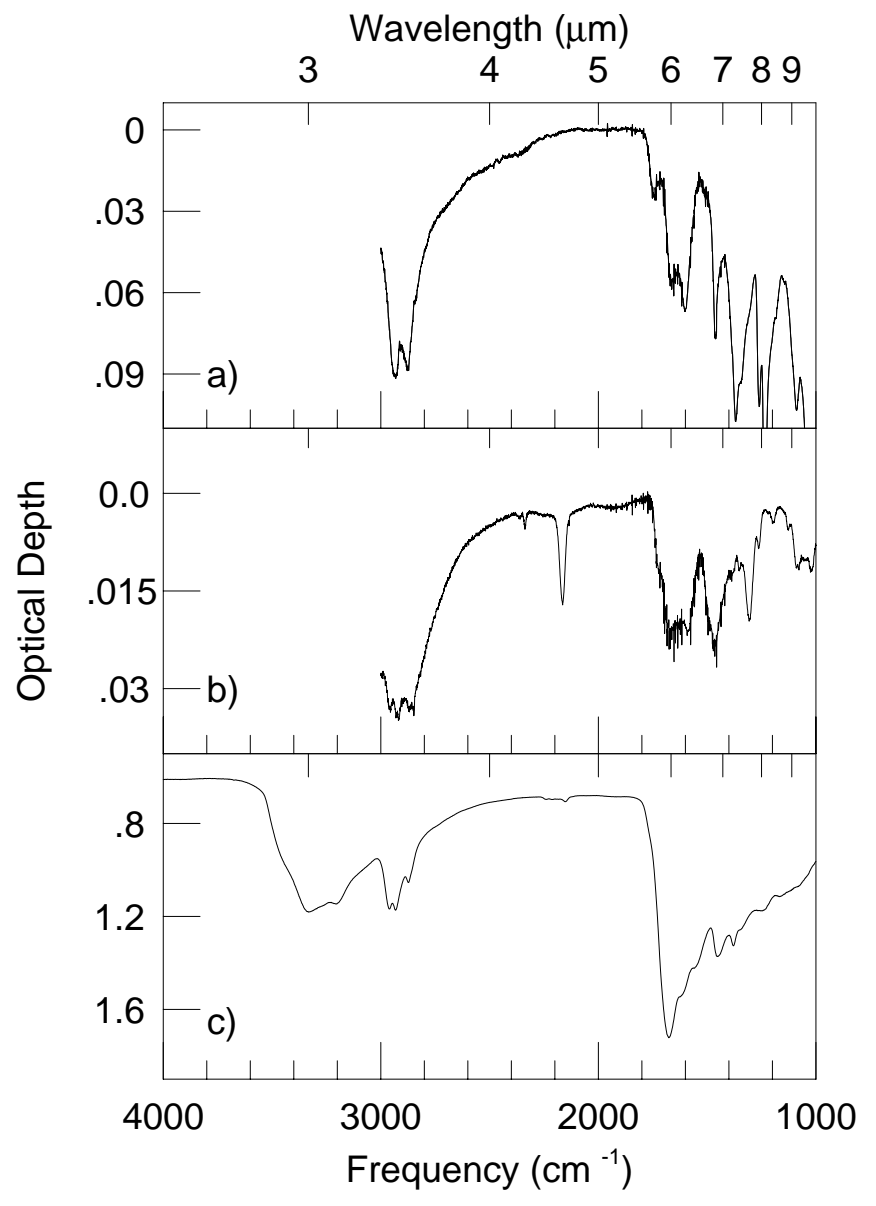

FIG. 6.-The $4000-1000 \mathrm{~cm}^{-1}(2.5-10 \mu \mathrm{m})$ spectrum of the residue materials produced by the UV irradiation of various interstellar ice analogs as described in $\S 4.1$. (a) $\mathrm{H}_{2} \mathrm{O}: \mathrm{CH}_{3} \mathrm{OH}: \mathrm{NH}_{3}: \mathrm{CO}(100: 50: 10: 10)$ Allamandola et al. (1988), Bernstein et al. (1995); (b) $\mathrm{H}_{2} \mathrm{O}: \mathrm{CH}_{3} \mathrm{OH}: \mathrm{NH}_{3}: \mathrm{CO}: \mathrm{C}_{3} \mathrm{H}_{8} \quad(100: 50: 10: 10: 10)$ Allamandola et al. (1988), (c) $\mathrm{H}_{2} \mathrm{O}: \mathrm{CO}: \mathrm{NH}_{3}: \mathrm{C}_{2} \mathrm{H}_{6}(5: 2: 2: 1)$ (Greenberg et al. 1995).

with the even broader acidic $\mathrm{OH}$ absorption. The $\mathrm{OH}$ and $\mathrm{NH}$ bending vibrations corresponding to these species likely contribute to the absorptions at about 1600,1380 , 1250, and $1000 \mathrm{~cm}^{-1} \quad(6.25,7.25,8.0$, and $10 \mu \mathrm{m}$, respectively). The two bands near 1750 and $1660 \mathrm{~cm}^{-1}(5.7$ and $6.0 \mu \mathrm{m}$, respectively) likely arise from the carbonyl CO stretch. One is probably associated with the carboxylic acid. The presence of two features in this region indicates another type of carbonyl is present as well, such as that found in aldehydes, ketones, or amides (see Fig. 7 for chemical configurations of carbonyl groups).

Figures $5 a$ and $6 a$ show that a strong, double-peaked aliphatic $\mathrm{CH}$ stretch band between 3000 and $2899 \mathrm{~cm}^{-1}$ is characteristic of this residue. The peak positions are consistent with $-\mathrm{CH}_{2}-$ groups only, in keeping with all of the residues made upon photolysis of mixed molecular ices containing methanol as the hydrocarbon source (Allamandola et al. 1988; Bernstein et al. 1995). Bernstein et al. (1995) have shown this spectral structure arises from hexamethylenetetramine (HMT, $\mathrm{C}_{6} \mathrm{H}_{12} \mathrm{~N}_{4}$ ). The only aliphatic groups in the HMT molecule are $-\mathrm{CH}_{2}-$ groups. The observation that the $\mathrm{CH}$ stretch band is double-peaked rather than split into subpeaks corresponding to $-\mathrm{CH}_{3}$ and $\mathrm{CH}_{2}-$ groups indicates HMT is an important constituent of the residue. The corresponding $-\mathrm{CH}_{2}-$ deformation vibrations 


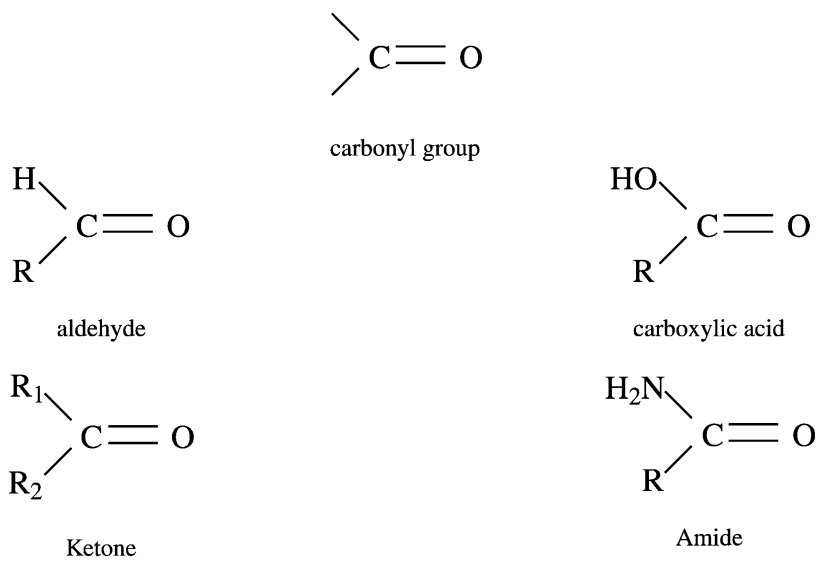

$\mathrm{R}, \mathrm{R}_{1}, \mathrm{R}_{2}=$ other chemical subgroups

FIG. 7.-Carbonyl structure and several different types of compounds in which it is found.

should give rise to a feature at about $1465 \mathrm{~cm}^{-1}(6.8 \mu \mathrm{m})$, precisely where a narrow band falls. Much of the sharp structure shortward of about $1500 \mathrm{~cm}^{-1}(6.6 \mu \mathrm{m})$ in this spectrum also arises from HMT (Bernstein et al. 1994, 1995). These bands are superposed on other residue features.

\subsubsection{Residue from $\mathrm{H}_{2} \mathrm{O}: \mathrm{CH}_{3} \mathrm{OH}: \mathrm{CO}: \mathrm{NH}_{3}: \mathrm{C}_{3} \mathrm{H}_{8}$ Irradiated Ice \\ 4.1.2.1. Material Preparation}

This organic residue is produced by the in situ, vacuum ultraviolet photolysis of the mixed molecular ice $\mathrm{H}_{2} \mathrm{O}: \mathrm{CH}_{3} \mathrm{OH}: \mathrm{CO}: \mathrm{NH}_{3}: \mathrm{C}_{3} \mathrm{H}_{8} \quad(100: 50: 10: 10: 10)$ after deposition onto a substrate at $10 \mathrm{~K}$. The lamp used is the same as that described in $\S$ 4.1.1. In this case however, the spectrum is of the residual material which remains on the substrate after the irradiated ice has been warmed up to 200 $\mathrm{K}$ (as opposed to room temperature) and then cooled to 10 $\mathrm{K}$ under vacuum. Allamandola et al. (1988) have reported this spectrum.

\subsubsection{Infrared Properties}

The IR spectrum of this material is shown in Figures $5 b$ and $6 b$. As with the previous spectrum, the detector failed at frequencies higher than about $3000 \mathrm{~cm}^{-1}$. The sloping baseline with onset near $2400 \mathrm{~cm}^{-1}$ is again indicative of carboxylic acids and hydrogen bonded $\mathrm{OH}$ and $\mathrm{NH}$ groups. There are several notable differences between the spectra in Figures $6 a$ and $6 b$. The $\mathrm{CH}$ stretch centered near $2900 \mathrm{~cm}^{-1}$ exhibits more substructure, there is a prominent feature near $2150 \mathrm{~cm}^{-1}(4.65 \mu \mathrm{m})$, and the pattern between 1800 and $1000 \mathrm{~cm}^{-1}$ is substantially different. These differences stem in part from the fact that this material has only been warmed to $200 \mathrm{~K}$, leaving species present which add structure to the spectrum. The other difference arises, of course, from the inclusion of propane $\left(\mathrm{C}_{3} \mathrm{H}_{8}\right)$ in the starting ice mixture. The laboratory spectrum reveals the following.

First, the prominent band centered at $2165 \mathrm{~cm}^{-1}$ arises from a nitrogen-and-carbon containing carrier, often referred to as $\mathrm{XCN}$, now $\mathrm{OCN}^{-}$. In all photolysis warm-up experiments this band disappears quickly above $250 \mathrm{~K}$ (d'Hendecourt et al. 1986; Allamandola et al. 1988; Bern- stein et al. 1995). A thorough discussion of this feature is presented in Demyk et al. (1998), Pendleton et al. (1999), Palumbo et al. (2000a, 2000b), and Bernstein et al. (2000).

The strong, broad complex centered near $1600 \mathrm{~cm}^{-1}$ is likely an overlap between $\mathrm{OH}$ bending modes with a carbonyl (see Fig. 7) stretch possibly arising from carboxylic acids, amides, ketones, esters, and alcohols. The prominent absorption peak near $1480 \mathrm{~cm}^{-1}$ is likely due to $\mathrm{CH}$ deformation modes of the aliphatic groups which give rise to the dominant $\mathrm{CH}$ stretch absorption near $2900 \mathrm{~cm}^{-1}$. The moderately broad band centered near $1050 \mathrm{~cm}^{-1}$ is consistent with the presence of alcohols $(-\mathrm{OH})$.

Figures $5 b$ and $6 b$ indicate the presence of $-\mathrm{CH}_{3}$ and $-\mathrm{CH}_{2}-$ groups in the $\mathrm{CH}$ stretch region. The presence of $-\mathrm{CH}_{3}$ in this ice residue, but not in the residue shown in Figure $5 a$ is reasonable in view of the inclusion here of the aliphatic hydrocarbon propane $\left(\mathrm{C}_{3} \mathrm{H}_{8}\right)$ in the initial mixture. Thus, instead of a residue dominated by HMT, this residue reflects the more typical aliphatic character as evidenced by the specific absorptions centered near 2960, 2920, 2870 , and $2855 \mathrm{~cm}^{-1}$, which are clear signatures of both $-\mathrm{CH}_{3}$ and $-\mathrm{CH}_{2}-$ groups. Further, absorption at the expected deformation mode positions near 1460 and 1370 $\mathrm{cm}^{-1}$ is present. The prominence of the $1460 \mathrm{~cm}^{-1}$ feature indicates the aliphatic component is $-\mathrm{CH}_{3}$ rich.

\subsubsection{Residue from Ice Irradiated by Ultraviolet Light in the Laboratory Followed by Irradiation in Space}

4.1.3.1. Material Preparation

Residues similar to those shown in Figures $5 a$ and $5 b$ (but with different starting compositions) were subsequently exposed to additional irradiation in space when they were flown aboard the Exobiology Radiation Assembly (ERA) platform on the EURECA satellite (Innocenti \& Mesland 1995). Ultraviolet irradiation and subsequent warm-up of a variety of ice mixtures including $\mathrm{H}_{2} \mathrm{O}, \mathrm{CO}, \mathrm{NH}_{3}, \mathrm{CH}_{4}$, $\mathrm{CH}_{3} \mathrm{OH}$, and $\mathrm{C}_{2} \mathrm{H}_{6}$ produced the original samples. The ices were deposited at $10 \mathrm{~K}$ with simultaneous vacuum UV irradiation for 22-68 hours. The UV radiation consisted of an intense narrow line at $\mathrm{Ly} \alpha(1216 \AA)$ and a several hundred $\AA$ wide band peaking near $1600 \AA$. The spectrum analyzed here is of the residue initially produced from an $\mathrm{H}_{2} \mathrm{O}: \mathrm{CO}: \mathrm{NH}_{3}: \mathrm{C}_{2} \mathrm{H}_{6}(5: 2: 2: 1)$ mixed molecular ice as reported by Mendoza-Gomez (1992). Residues were then sealed and carried into space where they remained in orbit at an altitude of $500 \mathrm{~km}$ for 11 months. During this time, they were exposed to the full solar spectrum for a total of 4 months. Although the samples appeared the same in terms of morphological structure upon their return, their visual color changed.

\subsubsection{Infrared Properties}

The infrared spectrum of this material is shown in Figures $5 c$ and $6 c$. As deduced from the two spectra above (Figs. $5 a$ and $5 b$ ), the strong, broad absorption seen here between 3550 and $2400 \mathrm{~cm}^{-1}$ implies the presence of alcohols $(-\mathrm{OH})$, carboxylic acid groups $(-\mathrm{COOH})$, possibly some trapped $\mathrm{H}_{2} \mathrm{O}$, and amines $\left(-\mathrm{NH}_{2}\right)$. A strong carbonyl $\mathrm{CO}$ stretch band near $1700 \mathrm{~cm}^{-1}$ could be hidden in the deep, broad band extending from $1750 \mathrm{~cm}^{-1}$ to beyond $1000 \mathrm{~cm}^{-1}$, consistent with the carboxylic acid assignment (see further discussion below). There may also be contributions from $\mathrm{OH}$ bending modes in water, acids, and alcohols. The structure in the band between 3000 and $2800 \mathrm{~cm}^{-1}$ (Fig. 5c) and the expected $\mathrm{OH}$ deformation modes (Fig. $6 c$ ) 
show the presence of aliphatic $-\mathrm{CH}_{3}$ and $-\mathrm{CH}_{2}-$ groups. The $\mathrm{CH}$ deformation vibrations are evident near 1465 and $1370 \mathrm{~cm}^{-1}$ in the broad, low-frequency envelope.

A few specific points can be made regarding the very strong feature starting at $1750 \mathrm{~cm}^{-1}$. First, the strong onset and clear subpeak at about $1700 \mathrm{~cm}^{-1}$ is evidence for a carbonyl group, consistent with the carboxylic acid assignment, possibly implicating aldehydes, ketones, and amides as well (Fig. 7). Other substructure (near 1050 and 1250 $\mathrm{cm}^{-1}$ ) is consistent with an $\mathrm{OH}$ bend and single bonded $\mathrm{CO}$ stretch. The IR spectrum indicates that additional subgroups also comprise this complex material, although severe band overlap precludes further specificity. A discussion of the astrophysical significance of this material can be found in Greenberg et al. (1995), Greenberg et al. (2000), and Greenberg \& Munoz Caro (2000).

\subsection{Residues Produced in Ices Bombarded by Energetic Particles}

4.2.1. Residue from Ammonia $\left(\mathrm{NH}_{3}\right)$ Containing Ice Bombarded by Energetic Particles

4.2.1.1. Material Preparation

This residue is produced by the proton bombardment of a $\mathrm{CH}_{4}: \mathrm{NH}_{3}$ ice mixture $(\sim 2: 1)$ condensed onto a substrate at $17 \mathrm{~K}$. The ice sample was irradiated with $0.8 \mathrm{MeV}$ protons with a fluence of $1.5 \times 10^{15}$ protons $\mathrm{cm}^{-2}$, equivalent to a total calculated dose of $26 \mathrm{eV} / 16 \mathrm{amu}$. This spectrum of the residue, which remains after warm-up to $300 \mathrm{~K}$, has been kindly provided by Marla Moore.

\subsubsection{Infrared Properties}

The infrared spectrum of this material is shown in Figures $8 a$ and $9 a$, where it is clearly dominated by a strong aliphatic $\mathrm{CH}$ stretch between $3000-2800 \mathrm{~cm}^{-1}$ with substructure indicating both $-\mathrm{CH}_{3}$ and $-\mathrm{CH}_{2}-$ groups (2960 and $2930 \mathrm{~cm}^{-1}$ ), respectively. The lower frequency bands are all weaker than the $\mathrm{CH}$ stretch, with broad subpeaks at about 1650 and $1580 \mathrm{~cm}^{-1}$, implicating the presence of aldehydes and ketones. Somewhat sharper bands appearing near 1470,1380 , and possibly $1320 \mathrm{~cm}^{-1}$, are consistent with the aliphatic $\mathrm{CH}$ deformations expected from the same groups that produce the aliphatic $\mathrm{CH}$ stretch complex between $3000-2800 \mathrm{~cm}^{-1}$. Since the intrinsic strength of the carbonyl band near $1600 \mathrm{~cm}^{-1}$ is typically 10 or more times greater than the intrinsic strength of the aliphatic $\mathrm{CH}$ deformations (which are 3 times stronger in this material), the broad, double-peaked absorption centered near $1600 \mathrm{~cm}^{-1}$ suggests that the aldehydic and ketonic species are present at most at about the three percent level. The dominance of the $\mathrm{CH}$ stretch band over the $\mathrm{CH}$ deformation feature is a very strong indicator that the aliphatic component of this material has a lot of pure alkane $\left[\mathrm{H}_{3} \mathrm{C}-\left(\mathrm{CH}_{2}\right)_{n}\right]$ character. This is substantially different from previous examples of irradiated ices (shown in Figs. $5 a, 5 b, 6 a$, and $6 b$ ) in which the aliphatic $\mathrm{CH}$ stretch bands were comparable to those of the $\mathrm{CH}$ deformations.

The weak band at about $2150 \mathrm{~cm}^{-1}$ is consistent with a nitrile, $(-\mathrm{C} \equiv \mathrm{N})$ or isonitrile $(\mathrm{C} \equiv \mathrm{N}-)$ (e.g., Bernstein et al. 1997; d'Hendecourt et al. 1986). The other ice residue spectra shown in Figures $6 b, 6 c, 9 b$, and the hydrogenated amorphous carbon spectrum shown in Fig $11 d$ are the only ones with absorption in the nitrile region. Energetic processing of nitrogen containing ices can yield an absorption band near $2165 \mathrm{~cm}^{-1}$ via either ion bombardment or UV

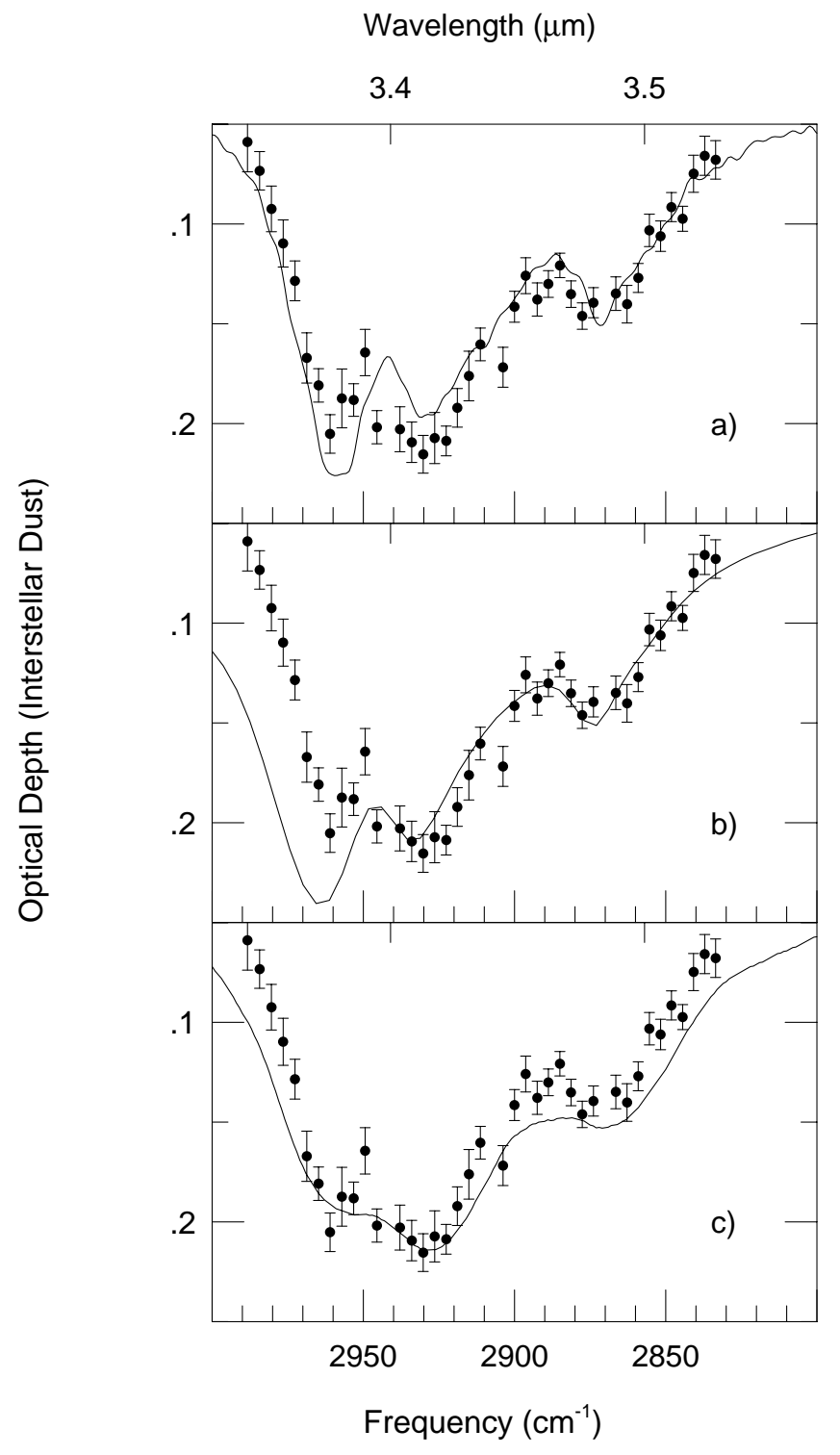

FIG. 8.-Comparison of the diffuse interstellar medium $3.4 \mu \mathrm{m}$ absorption features (points) to the corresponding band in the spectrum of the residue materials (lines) produced by the energetic particle bombardment of various interstellar ice analogs as described in $\S 4.2$. The Diffuse Interstellar Medium spectrum (GC IRS 6E) is taken from Pendleton et al. (1994). Laboratory spectra: (a) $\mathrm{CH}_{4}: \mathrm{NH}_{3}(\sim 2: 1)$ (M. Moore 2001, private communication); (b) $\mathrm{H}_{2} \mathrm{O}: \mathrm{N}_{2}: \mathrm{CH}_{4}(\sim 0.06: 1: 1)$ (M. Moore 2001, private communication); (c) $\mathrm{CH}_{4}$ (Strazzulla et al. 1991). The spectrum shown is of this residue material at room temperature after it has been exposed to the atmosphere.

photolysis (Pendleton et al. 1999 and others). Discussion of the effects of ion bombardment versus UV photolysis as energetic processors of ice analogs is presented in Gerakines, Moore, \& Hudson (2000) and Moore, Hudson, \& Gerakines (2000).

\subsubsection{Residue from a Nitrogen $\left(\mathrm{N}_{2}\right)$ Containing Ice Bombarded by Energetic Particles \\ 4.2.2.1. Material Preparation}

This residue is produced by the proton bombardment of an $\mathrm{H}_{2} \mathrm{O}: \mathrm{N}_{2}: \mathrm{CH}_{4}$ ice mixture $(0.06: 1: 1)$ condensed onto a substrate at $17 \mathrm{~K}$. The ice sample was irradiated with 1 $\mathrm{MeV}$ protons with a fluence of $1.8 \times 10^{15}$ protons $\mathrm{cm}^{-2}$, equivalent to a total calculated dose of $28 \mathrm{eV} / 16 \mathrm{amu}$. The 


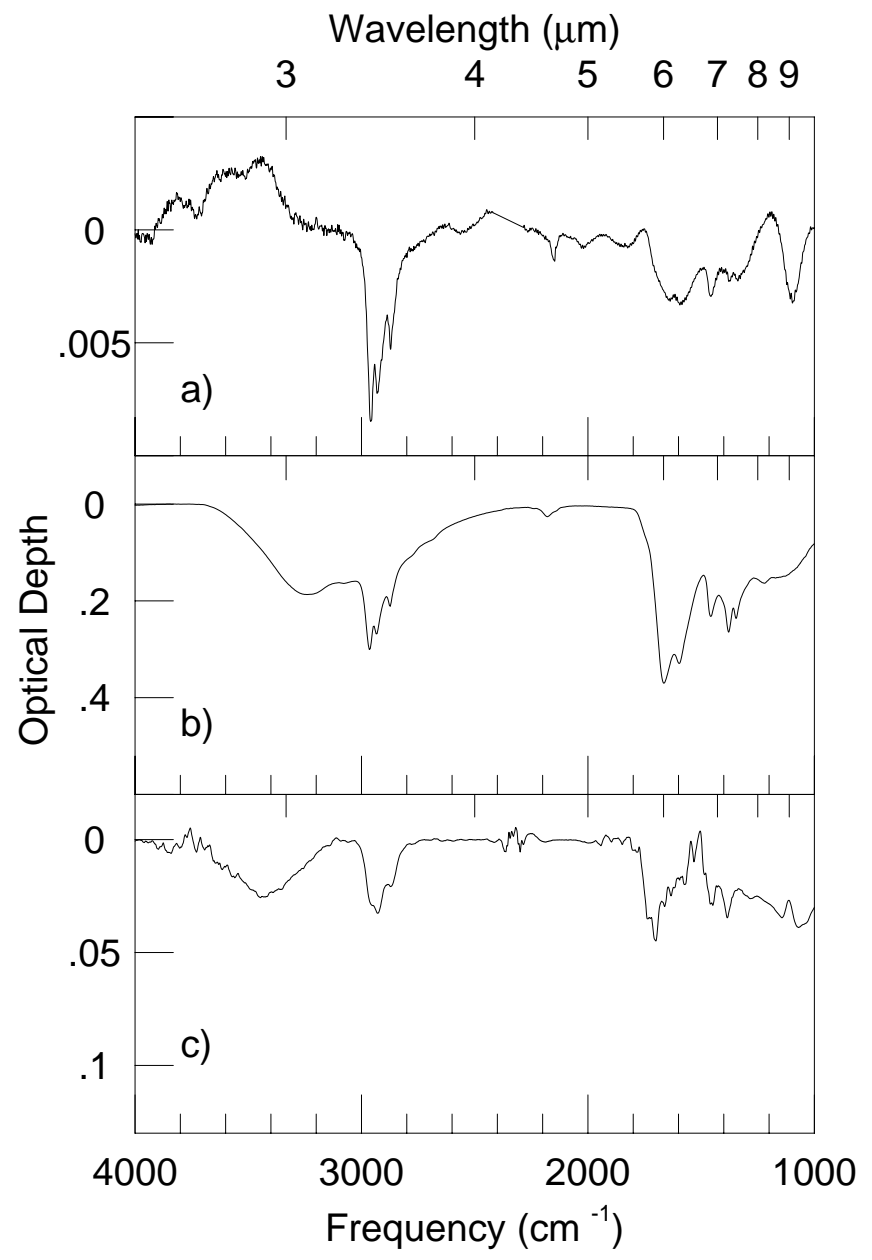

FIG. 9. - The $4000-1000 \mathrm{~cm}^{-1}(2.5-10 \mu \mathrm{m})$ spectrum of the residue materials produced by the energetic particle bombardment of various interstellar ice analogs as described in $\S 4.2$. (a) $\mathrm{CH}_{4}: \mathrm{NH}_{3}$ (2:1) (M. Moore 2000, private communication); (b) $\mathrm{H}_{2} \mathrm{O}: \mathrm{N}_{2}: \mathrm{CH}_{4}(\sim 0.06: 1: 1)$ (M. Moore 2000, private communication); (c) $\mathrm{CH}_{4}$, Strazzulla et al. 1991). The spectrum shown is of this residue material at room temperature after it has been exposed to the atmosphere. The feature near $2340 \mathrm{~cm}^{-1}$ is due to atmospheric carbon dioxide.

spectrum of the residue remaining after warm-up to $300 \mathrm{~K}$ has been kindly provided by Marla Moore (2000, private communication). Details of the procedures used can be found in Moore et al. (2000) and Hudson \& Moore (2000).

\subsubsection{Infrared Properties}

The infrared spectrum of this material is shown in Figures $8 b$ and $9 b$. Comparing this spectrum to that shown in Figure $9 a$ reveals that this material is substantially different, with oxygen playing a far more important role. First, the moderate, broad absorption between about 3800 and $2600 \mathrm{~cm}^{-1}$ indicates $\mathrm{OH}$, and the strong doublet feature centered near $1650 \mathrm{~cm}^{-1}$ is indicative of carbonyls. Taken together, these bands indicate the presence of carboxylic acids and other species such as aldehydes and/or ketones. There is also a moderately strong, very broad absorption at the low frequency limit of the spectrum consistent with oxygen in the form of the $\mathrm{CO}$ linkage in ethers and alcohols overlapping with $\mathrm{OH}$ bending vibrations.

The material exhibits a moderately strong aliphatic $\mathrm{CH}$ stretch absorption between $3000-2800 \mathrm{~cm}^{-1}$ with substructure showing the presence of both $-\mathrm{CH}_{3}$ and $-\mathrm{CH}_{2}-$ groups. The lower frequency bands are all stronger than or comparable to the $\mathrm{CH}$ stretch, unlike the previous example (Fig. 9a). The material in Figure $9 b$ shows a triplet in the $\mathrm{CH}$ deformation region, with clear components at 1470 , 1390 , and $1350 \mathrm{~cm}^{-1}$ very close to the corresponding bands in the previous case.

The comparable strengths of the aliphatic $\mathrm{CH}$ stretches to the deformation bands indicates these materials are not pure alkanes $\left[\mathrm{H}_{3} \mathrm{C}-\left(\mathrm{CH}_{2}\right)_{n}-\right]$ in character, but include other chemical entities within their structures in close proximity to the $-\mathrm{CH}_{3}$ and $-\mathrm{CH}_{2}-$ groups. The comparable strengths of the aliphatic stretches to the deformation bands, plus the differences in low frequency substructure between the two spectra (Figs. 9a and 9b), imply that oxygen is incorporated intramolecularly within the residue. Finally, the weak band close to $2160 \mathrm{~cm}^{-1}$ is indicative of a nitrile or perhaps isonitrile as discussed in $\S \S$ 4.1.2 and 4.2.1.

\subsubsection{Residue from Methane $\left(\mathrm{CH}_{4}\right)$ Ice Bombarded by Energetic Particles \\ 4.2.3.1. Material Preparation}

This residue was produced by irradiating methane $\left(\mathrm{CH}_{4}\right)$ ice, with $75 \mathrm{keV}$ protons at a dose of $266 \mathrm{eV} / 16 \mathrm{amu}$. The protons were accelerated with a $400 \mathrm{kV}$ ion implanter. The spectrum shown is of the residue material at room temperature after it has been exposed to the atmosphere. This spectrum was kindly provided by Giovanni Strazzulla, and experimental details can be found in Strazzulla, Baratta, \& Magazzu (1991).

\subsubsection{Infrared Properties}

The infrared spectrum of this material is shown in Figures $8 c$ and $9 c$. The extent of the moderately strong broad absorption between roughly 3650 and $3200 \mathrm{~cm}^{-1}$ indicates extensive $\mathrm{OH}$ hydrogen bonding typical of alcohols, rather than acids, since the low frequency limit stops near $3100 \mathrm{~cm}^{-1}$, not $2500 \mathrm{~cm}^{-1}$. Further, the absence of the acidic $\mathrm{OH}$ stretch, but the presence of a strong carbonyl absorption peaking near $1700 \mathrm{~cm}^{-1}$, implies that ketonic or aldehydic groups are present. Given that the intrinsic strength of the hydrogen-bonded $\mathrm{OH}$ stretch and carbonyl $\mathrm{C}=\mathrm{O}$ stretch is at least 5-10 times stronger than the intrinsic CH stretch (e.g., Allamandola 1984), these groups are probably present at about the $10 \%$ level in this residue. Aliphatic $-\mathrm{CH}_{3}$ and $-\mathrm{CH}_{2}-$ groups are also important in this material as shown by the strong band between 3000 and $2800 \mathrm{~cm}^{-1}$, comparable in strength to the $\mathrm{OH}$ stretch band. The corresponding aliphatic $\mathrm{CH}$ deformation bands are clearly present and fall near 1460 and $1390 \mathrm{~cm}^{-1}$ in this material.

\subsection{Carbonaceous Residues Produced from Carbon Rods and Plasmas \\ 4.3.1. Carbonaceous Particulates Collected from a Heated Carbon Rod \\ 4.3.1.1. Material Preparation}

Subliming carbon from a heated graphite rod produces this material. The carbon vapor is quenched in a $10 \mathrm{mb}$ $\mathrm{Ar} / \mathrm{H}_{2}$ atmosphere and the resulting particles are collected on a surface. Production, analysis, and spectroscopic properties of this nanoparticulate material, labeled $\mathrm{H} 50$, are discussed in Schnaiter et al. (1998, 1999), and Henning \& Schnaiter (1999). 
4.3.1.2. Infrared Properties

The infrared spectrum of the material shown in Figures $10 a$ and $11 a$ demonstrates a weak $\mathrm{OH}$ stretch band between 3600 and $3100 \mathrm{~cm}^{-1}$. However, since the intrinsic $\mathrm{OH}$ band intensity for hydrogen bonded $\mathrm{OH}$ is exceptionally strong (e.g., Allamandola 1984 and references therein), this weak feature indicates low levels of oxygen. Similarly, the moderate carbonyl absorption between about 1800 and 1700 $\mathrm{cm}^{-1}$ is also consistent with the presence of minor amounts

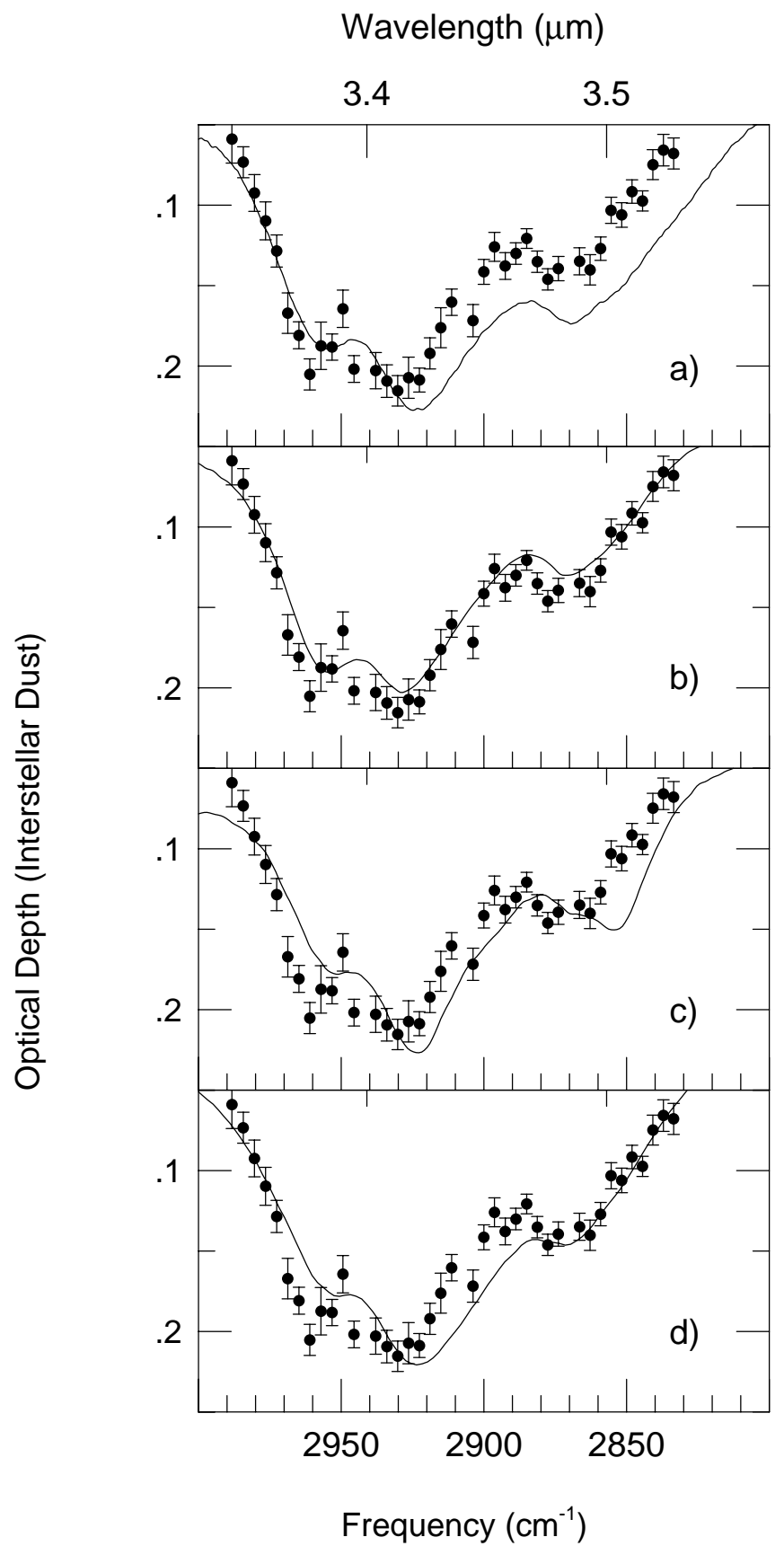

Fig. 10.-Comparison of the diffuse interstellar medium $3.4 \mu \mathrm{m}$ absorption features (points) to the corresponding band (lines) in the spectrum of the reactants produced from the various carbonaceous reactants described in $\S$ 4.3. The Diffuse Interstellar Medium spectrum is taken from Pendleton et al. (1994). Laboratory spectra: (a) Heated carbon rod, Schnaiter et al (1999); (b) Hydrogenated, laser desorbed, amorphous carbon, Mennella et al. (1999); (c) Aromatic plasma (AC electric discharge), Lee \& Wdowiak (1993); (d) $\mathrm{CH}_{4}$ plasma (DC electric discharge), Furton et al. (1999).

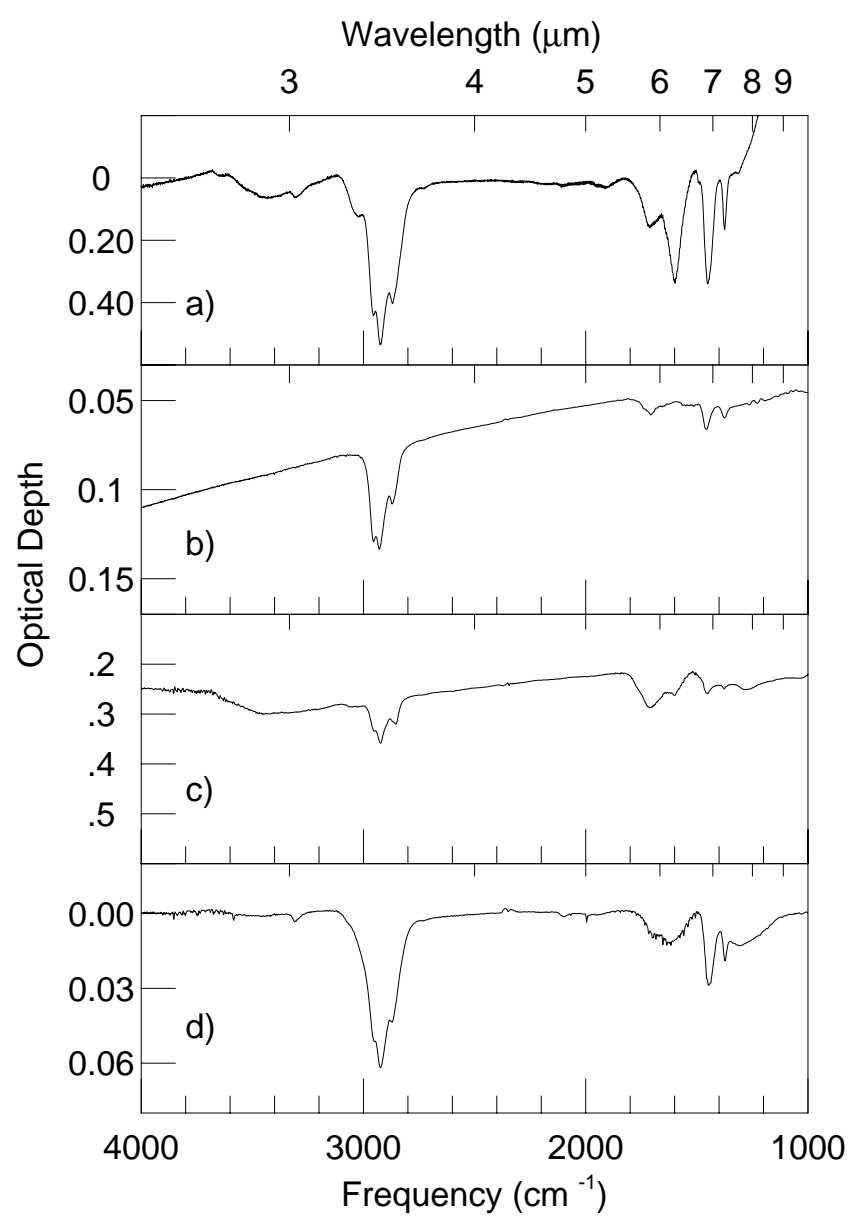

FIG. 11.-The $4000-1000 \mathrm{~cm}^{-1}(2.5-10 \mu \mathrm{m})$ spectrum of the materials produced from various carbonaceous materials as described in $\S 4.3$. (a) Heated carbon rod, Schnaiter et al (1999); (b) Hydrogenated, laser desorbed, amorphous carbon, Mennella et al. (1999); (c) Aromatic plasma (AC electric discharge) Lee \& Wdowiak (1993); $(d) \mathrm{CH}_{4}$ plasma (DC electric discharge) Furton et al. (1999).

of oxygen as this vibration also gives rise to an intrinsically very strong band. The spectrum shown in Figure $11 a$ has had the high frequency scattering continuum removed from that shown by Schnaiter et al. (1998) in their Figure 6.

A very strong aliphatic $\mathrm{CH}$ stretch band that shows the presence of $-\mathrm{CH}_{2}-$ and $-\mathrm{CH}_{3}$ groups dominates the spectrum of this material. The weak, but clear, shoulder peaking near $3030 \mathrm{~cm}^{-1}$ indicates that some alkenes $\left(\mathrm{C}_{n} \mathrm{H}_{2 n}\right)$ and aromatic hydrocarbons are present as well, and the small peak near $3300 \mathrm{~cm}^{-1}$ is suggestive of an alkyne $(\equiv \mathrm{C}-\mathrm{H}$ ) hydrocarbon stretch (Fig. 2). The dominance of the spectrum by the strong aliphatic $\mathrm{CH}$ stretch and the clear aliphatic $\mathrm{CH}$ deformation bands (near 1480 and 1390 $\mathrm{cm}^{-1}$ ) indicate that alkanes are an important hydrocarbon form of this material. However, the comparable strengths of the aliphatic $\mathrm{CH}$ stretch and deformation bands indicate the residue is not made up of pure alkanes, but rather contains alkane chains a few carbon atoms long bonded to other groups including alkenes, alkynes, and aromatics. Given that the intrinsic strength of the aromatic $\mathrm{CH}$ stretch at $3030 \mathrm{~cm}^{-1}$ is roughly 10 times weaker than the intrinsic strength of the aliphatic $\mathrm{CH}$ stretch centered near 2900 $\mathrm{cm}^{-1}$, the relative strengths of these two bands indicates a comparable numbers of aromatic and aliphatic $\mathrm{CH}$ bonds 
in this material. Further evidence of aromatic material in this sample arises from the prominent $1600 \mathrm{~cm}^{-1}$ band. The three-peaked profile of the aliphatic $\mathrm{CH}$ stretch absorption band is consistent with this explanation as well (e.g., Pendleton et al. 1994; Sandford et al. 1991).

\subsubsection{Carbonaceous Particulates from Laser Ablated Carbon Rods Plus Hydrogenation by Exposure to Hydrogen Atoms}

4.3.2.1. Material Preparation

These carbon grains, called ACARL, were produced by laser sputtering of an amorphous carbonaceous material under a $10 \mathrm{mb}$ Ar atmosphere. The samples were then exposed to $\mathrm{H}$ atoms $\left(6.9 \times 10^{19}\right.$ atoms $\left.\mathrm{cm}^{-2}\right)$. Details can be found in Mennella et al. (1999).

\subsubsection{Infrared Properties}

The infrared spectrum of this material is shown in Figures $10 b$ and $11 b$. The infrared spectrum is clearly dominated by alkane bonding within the structure. The clean aliphatic $\mathrm{CH}$ stretch between 3000 and $2800 \mathrm{~cm}^{-1}$ and corresponding deformations at about 1470 and $1380 \mathrm{~cm}^{-1}$, along with the relative intensities of these features make this a clear-cut conclusion. The very weak carbonyl band near $1710 \mathrm{~cm}^{-1}$ indicates that there is a very small trace of oxygen in this material. The lack of 3030 and $1600 \mathrm{~cm}^{-1}$ bands implies there is little aromatic character evident although the weak, broad feature between about 1600 and $1100 \mathrm{~cm}^{-1}$ is consistent with the presence of some aromatic material. Note the striking similarity between this spectrum and that in Figure $12 c$ (plasma ablated graphite; Scott, Duley, \& Pinho 1997).

\subsubsection{Carbonaceous Material from an Electric Discharge through an Aromatic/Hydrogen Vapor}

4.3.3.1. Material Preparation

This is the material which condenses on the chamber walls when a $9400 \mathrm{~V}$ AC electric discharge is passed through a gaseous mixture of hydrogen and the aromatic molecule naphthalene $\left(\mathrm{C}_{10} \mathrm{H}_{8}\right)$. Details can be found in Lee \& Wdowiak (1993).

4.3.3.2. Infrared Properties

The infrared spectrum of this material is shown in Figures $10 c$ and $11 c$. The very weak broad band centered about $3400 \mathrm{~cm}^{-1}$ indicates the presence of some hydrogenbonded $\mathrm{OH}$, but given the exceptional intrinsic strength of this mode, oxygen is but a trace component. The spectrum of this material is similar to that of the previous two materials discussed. It is dominated by the aliphatic $\mathrm{CH}$ stretch band with subpeaks near 2955, 2922, and 2852 $\mathrm{cm}^{-1}$. The corresponding $\mathrm{CH}$ bending vibrations at 1460 and $1380 \mathrm{~cm}^{-1}$ are also clearly evident.

There is also a weak aromatic $\mathrm{CH}$ stretch band at about $3030 \mathrm{~cm}^{-1}$. The corresponding aromatic CC stretch likely contributes to the $1610 \mathrm{~cm}^{-1}$ absorption, and the much broader band centered near $1300 \mathrm{~cm}^{-1}$ which spans the region from 1500 to $1100 \mathrm{~cm}^{-1}$ is consistent with the aromatic $\mathrm{CC}$ stretching and $\mathrm{CH}$ in-plane bending-combination vibration. Again, given that the intrinsic strength of the aromatic $\mathrm{CH}$ stretch at $3030 \mathrm{~cm}^{-1}$ is about 10 times weaker than the intrinsic strength of the aliphatic $\mathrm{CH}$ stretch centered near $2900 \mathrm{~cm}^{-1}$, the relative strengths of these two bands indicates there are comparable amounts of aromatic and aliphatic $\mathrm{CH}$ bonds in this material.

There is also a clear-cut carbonyl stretch at $1700 \mathrm{~cm}^{-1}$. Considering the unusual intrinsic strength of the $\mathrm{CO}$ band, one concludes that oxygen is not a major component of this material.

The weak $\mathrm{OH}$, carbonyl and aromatic bands, and the stronger aliphatic $\mathrm{CH}$ stretch to $\mathrm{CH}$ deformation absorptions, indicate the residue is a hydrocarbon-rich material with aliphatic and aromatic character, in which the alkane component is dominant.

\subsubsection{Carbonaceous Material from an Electric Discharge through Methane \\ 4.3.4.1. Material Preparation}

This is the material that deposits out onto the walls when a pure gaseous methane stream is passed through a $1000 \mathrm{~V}$ DC electric discharge. The preparation of this material, which is referred to as an interstellar HAC analog, is described in Furton, Laiho, \& Witt (1999).

4.3.4.2. Infrared Properties

The infrared spectrum of this material is shown in Figures $10 d$ and $11 d$. While the spectrum of this material possesses some similarities to that just discussed and shown in Figures $10 c, 11 a, 11 b$, and $11 c$, some important differences are evident. The small $3300 \mathrm{~cm}^{-1}$ peak suggests alkynes ( $\equiv \mathrm{C}-\mathrm{H}$ stretch) are present, and the $2100 \mathrm{~cm}^{-1}$ band is evidence for nitriles $(-\mathrm{C} \equiv \mathrm{N})$.

The similarities with the spectra in Figures $11 a, 11 b$, and $11 c$ include the aliphatic $\mathrm{CH}$ stretch band complex peaking near $2922 \mathrm{~cm}^{-1}$, and the relative strength of the corresponding $\mathrm{CH}$ bending vibrations at 1450 and $1375 \mathrm{~cm}^{-1}$. Further, there is a similar, but less structured, absorption band centered near $1600 \mathrm{~cm}^{-1}$. The aliphatic component extends to $3100 \mathrm{~cm}^{-1}$, indicating unresolved overlap with an aromatic $\mathrm{CH}$ stretch at $3050 \mathrm{~cm}^{-1}$. The presence of aromatic material in this sample is supported by the unresolved absorption at $1600 \mathrm{~cm}^{-1}$ and the broad band under the aliphatic deformation bands extending from about 1500 to $1100 \mathrm{~cm}^{-1}$ which peaks near $1250 \mathrm{~cm}^{-1}$. There is also evidence for a carbonyl stretch peak at $1700 \mathrm{~cm}^{-1}$. Again, taking the unusual intrinsic strength of this band into account implies that oxygen is not a major component of this material.

\subsubsection{Carbonaceous Material from a Microwave Discharge through Methane-Unheated QCC}

4.3.5.1. Material Preparation

Passing methane through a microwave-powered discharge and capturing the condensate as a film deposited on the walls of the reaction chamber produces this material. The spectrum of this material, called filmy QCC (Quenched Carbonaceous Condensate), was kindly provided by S. Wada. The production details can be found in Sakata et al. (1983) and Sakata et al. (1987).

\subsubsection{Infrared Properties}

The infrared spectrum of this material is shown in Figures $12 a$ and $13 a$. The strongest feature in the overall spectrum of this material is the aliphatic $\mathrm{CH}$ stretch peaking near $2900 \mathrm{~cm}^{-1}$. The subpeak near $2920 \mathrm{~cm}^{-1}$ $\left(-\mathrm{CH}_{2}-\right.$ stretch) is about twice as deep as the shoulder near $2960 \mathrm{~cm}^{-1}\left(-\mathrm{CH}_{3}\right.$ stretch) showing that this material has about twice as many $-\mathrm{CH}_{2}-$ as $-\mathrm{CH}_{3}$ groups. The corresponding $\mathrm{CH}$ bending modes are clear at 1440, 1380, and $1310 \mathrm{~cm}^{-1}$, and the relative intensities of these to the $\mathrm{CH}$ stretch bands indicate that while the residue has alkanelike properties, other types of material are also an integral part of the composition. 


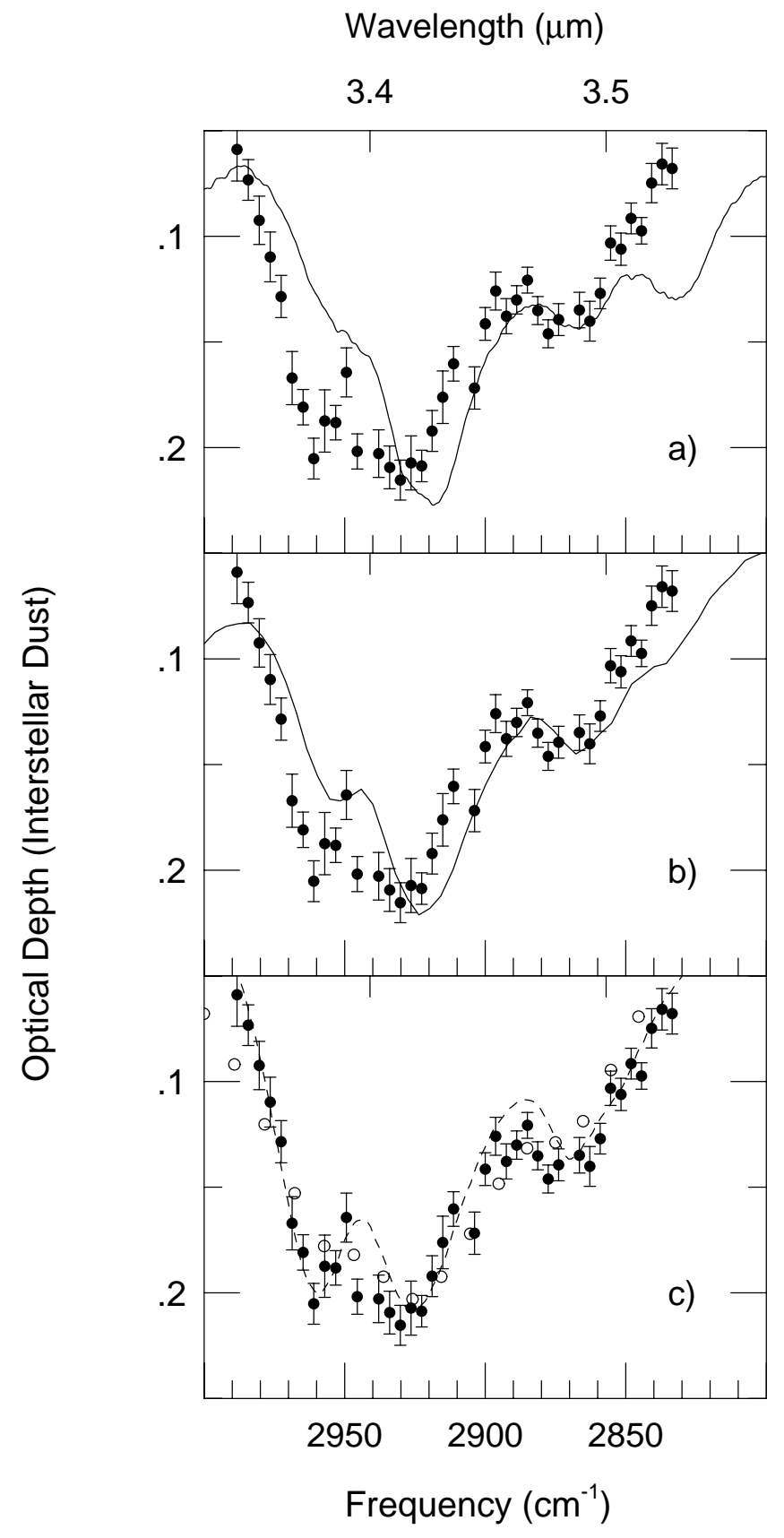

FIG. 12.-Comparison of the diffuse interstellar medium $3.4 \mu \mathrm{m}$ absorption features (points) to the corresponding band in the spectrum of the materials produced by the plasma discharge of various hydrocarbons (lines) as described in $\S 4.3$. The diffuse Interstellar Medium spectrum (GC IRS 6E) is taken from Pendleton et al. (1994). Laboratory spectra: (a) $\mathrm{CH}_{4}$ plasma (microwave) (S. Wada 2000, private communication, after Sakata \& Wada 1989); (b) $\mathrm{CH}_{4}$ plasma (microwave) residue (heated) (S. Wada 2000, private communication, after Sakata \& Wada 1989); (c) plasma ablated graphite in hydrogen (Scott et al. 1997). Open points correspond to material deposited at room temperature, and the dashed line corresponds to material deposited at $77 \mathrm{~K}$.

There is a prominent aromatic $\mathrm{CH}$ stretch band at 3000 $\mathrm{cm}^{-1}$, with the aromatic assignment supported by other bands. The distinct aromatic feature at $1600 \mathrm{~cm}^{-1}$ and the very broad aromatic component expected between about 1400 and $1100 \mathrm{~cm}^{-1}$ is also present underlying the $\mathrm{CH}$. Further, the weak band near $1900 \mathrm{~cm}^{-1}$ is also consistent with aromatic material and generally reflects ring substitut-

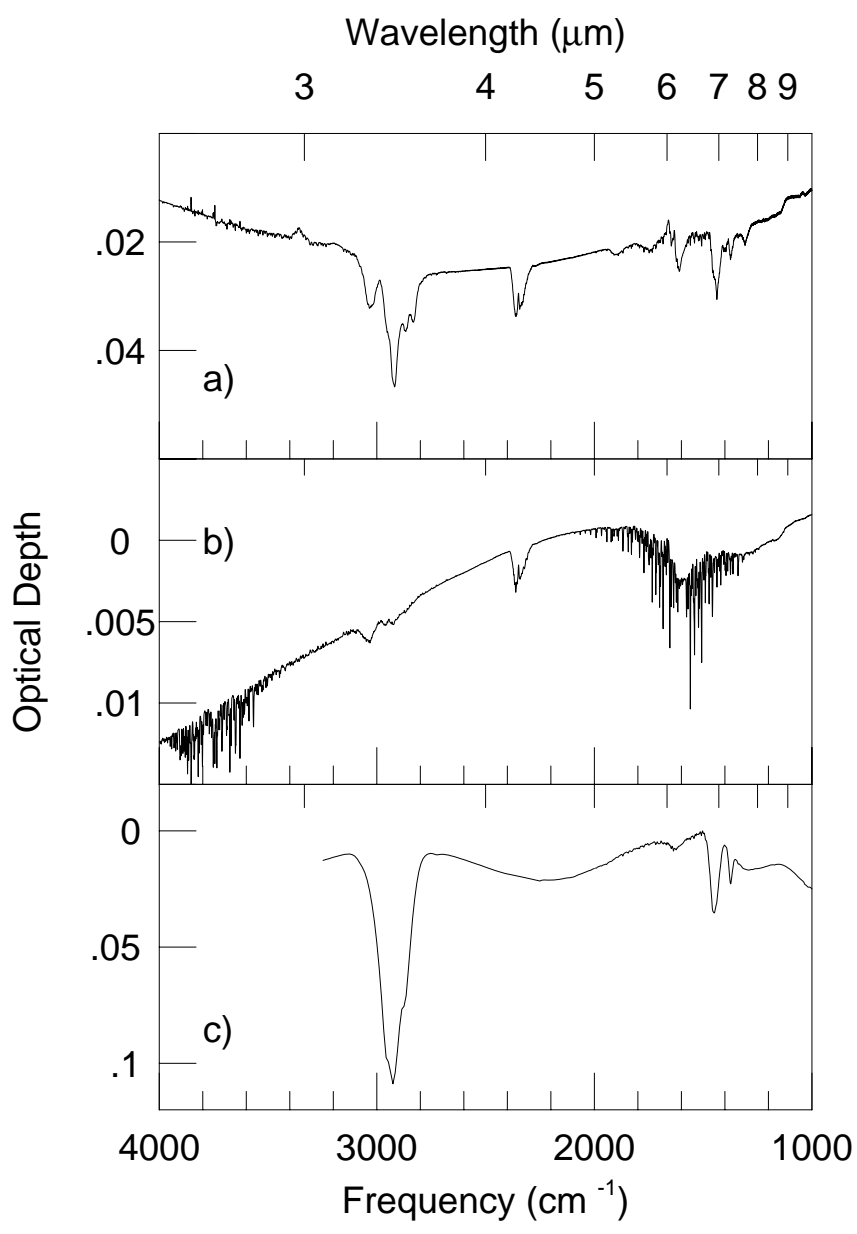

FIG. 13.-The $4000-1000 \mathrm{~cm}^{-1}(2.5-10 \mu \mathrm{m})$ spectrum of the materials produced by the plasma discharge of various hydrocarbons as described in $\S$ 4.3. (a) $\mathrm{CH}_{4}$ plasma (microwave) (S. Wada 2000, private communication, after Sakata \& Wada 1989); (b) $\mathrm{CH}_{4}$ plasma (microwave) residue heated to $400^{\circ} \mathrm{C}$ (courtesy of S. Wada 2000 , private communication, after Sakata \& Wada 1989); (c) plasma ablated graphite in hydrogen from Scott et al. (1997). The feature near $2340 \mathrm{~cm}^{-1}$ and sharp lines in frames $13 \mathrm{a}$ and $13 \mathrm{~b}$ are due to atmospheric $\mathrm{CO}_{2}$ and $\mathrm{H}_{2} \mathrm{O}$.

ion patterns. Given the aromatic to aliphatic $\mathrm{CH}$ intrinsic band strengths, the spectrum indicates that there are about twice as many aromatic $\mathrm{CH}$ bonds in this material as aliphatic $\mathrm{CH}$ bonds.

The absence of any absorption in the $\mathrm{OH}$ stretching region eliminates the possibility of an $\mathrm{OH}$ bend contributing to the $1600 \mathrm{~cm}^{-1}$ absorption, and the very weak band near $1700 \mathrm{~cm}^{-1}$ in the carbonyl region shows that oxygen is only present in trace amounts.

The double-peaked band near $2340 \mathrm{~cm}^{-1}$ arises from atmospheric $\mathrm{CO}_{2}$, not the residue. Similarly, the very weak, sharp features superposed between 4000 and $3200 \mathrm{~cm}^{-1}$ and 2000 and $1300 \mathrm{~cm}^{-1}$ on the spectrum arises from atmospheric water as discussed in the original work.

\subsubsection{Carbonaceous Material from a Microwave Discharge through Methane-Heated QCC \\ 4.3.6.1. Material Preparation}

Passing methane through a microwave-powered discharge and capturing the condensate as a film deposited on the walls of the reaction chamber produces this material. The spectra shown in Figures $12 b$ and $13 b$ of heated, filmy 
QCC (Quenched Carbonaceous Condensate) are of this material after it has been heated up to $450 \mathrm{C}$ (Sakata et al. 1987) and $400 \mathrm{C}$ (kindly provided by S. Wada), respectively. The production details can be found in Sakata et al. (1983) and Sakata et al. (1987).

\subsubsection{Infrared Properties}

The infrared spectrum of this material is shown in Figures $12 b$ and $13 b$. Comparing this to the spectrum of the unheated material shown in Figures $12 a$ and $13 a$ reflects the well-known aromatization process as an aliphatic-rich material is heated above about $300 \mathrm{C}$ (e.g., Low \& Mortera 1983; Dischler, Bubenzer, \& Koidl 1983). This is similar to the changes in the types of coal discussed by Papoular et al. (1996), Guillois et al. (1996), and others as analogs of interstellar dust. The aromatic $\mathrm{CH}$ stretch at $3000 \mathrm{~cm}^{-1}$ becomes stronger than the aliphatic $\mathrm{CH}$ stretch complex between 3000 and $2800 \mathrm{~cm}^{-1}$. The corresponding aliphatic $\mathrm{CH}$ bending modes are evident near 1430 and $1390 \mathrm{~cm}^{-1}$ in Figure $13 b$. However, these are essentially overwhelmed by the aromatic CC stretching band centered roughly at 1600 $\mathrm{cm}^{-1}$ and the broad aromatic $\mathrm{CC}$ stretching $\mathrm{CH}$ in-plane bending combination band between about 1500 and 1100 $\mathrm{cm}^{-1}$ which peaks near $1300 \mathrm{~cm}^{-1}$. The spectrum indicates that the overall nature of this material is aromatic with some aliphatics between aromatic moieties. The doublepeaked band near $2340 \mathrm{~cm}^{-1}$ arises from atmospheric $\mathrm{CO}_{2}$, not the residue, and the sharp structure between 4000 and $3200 \mathrm{~cm}^{-1}$ and 2000 and $1300 \mathrm{~cm}^{-1}$ superposed on the spectrum arises from atmospheric water as discussed in the original work.

\subsubsection{Hydrogenated Amorphous Carbon Films From Laser Ablation of Graphite in Hydrogen Atmosphere \\ 4.3.7.1. Material Preparation}

This hydrogenated amorphous carbon (HAC) was prepared using plasma deposition (Scott \& Duley 1996; Scott 1997; Duley et al. 1998) through the laser ablation of graphite in the presence of hydrogen. Condensation of the weakly ionized plasma containing carbon, hydrogen atoms, and a variety of hydrocarbon molecules results in a polymeric HAC solid with a band gap of $E_{g} \sim 2.5-3 \mathrm{eV}$. Two residues are considered here. One of the materials was deposited at room temperature (annealed) and the other was deposited at $77 \mathrm{~K}$ (unannealed). The density is higher and the hydrogen content lower for the sample deposited at room temperature.

\subsubsection{Infrared Properties}

The infrared spectrum of these materials is shown in Figures $12 c$ and $13 c$. The residue spectrum shown by open points in Figure $12 c$ is of the annealed HAC, while the dashed line corresponds to the unannealed HAC. Films deposited at $77 \mathrm{~K}$ provide a better fit to the $3.4 \mu \mathrm{m}$ bands, shown by the dashed line in Figure 12c. Unfortunately, the corresponding 5-10 $\mu \mathrm{m}$ data for those films were not available, so the 5-10 $\mu \mathrm{m}$ spectrum shown in Figure $12 c$ corresponds to the room temperature material shown by the open points in Figure 12c. Figure $13 c$ presents the longer wavelength spectrum for the room temperature, annealed HAC. The overall spectrum is dominated by the aliphatic $\mathrm{CH}$ stretch peaking near $2900 \mathrm{~cm}^{-1}$. The high frequency roll-off of this band is consistent with unresolved blending with aromatic $\mathrm{CH}$ absorption, implying that this material is comprised of aliphatic and aromatic $\mathrm{CH}$ groups. The struc- ture near 1460 and $1390 \mathrm{~cm}^{-1}$ corresponds to the aliphatic $\mathrm{CH}$ bends with the relative intensities implying that this material has a lot of aliphatic character. The small 1620 $\mathrm{cm}^{-1}$ band is likely due to an aromatic CC stretch, and the broad band between 1400 and $1200 \mathrm{~cm}^{-1}$, peaking near $1300 \mathrm{~cm}^{-1}$, is likely due to aromatic $\mathrm{CC}$ stretching and $\mathrm{CH}$ in-plane bending combination bands. Thus, although the spectrum appears largely aliphatic, this material contains an important aromatic fraction as well. Note the striking similarity between this spectrum and that in Figure $11 b$ ( 4.3.2; laser sputtering of an amorphous carbonaceous material from Mennella et al. 1999).

\subsubsection{Murchison Meteorite Extract}

4.3.8.1. Material Preparation

This is the sublimate from the acid and organic insoluble kerogen from the Murchison meteorite (DeVries et al. 1993; Kerridge et al. 1987). Fresh, internal samples of the Murchison meteorite were ground into a powder. Minerals and soluble organics were removed by repetitive acid/solvent extractions as described by Kerridge et al. (1987). After heating to $600^{\circ} \mathrm{C}$, the volatile component that sublimed onto the chamber walls was collected and analyzed. The spectrum shown here is of that sublimate.

\subsubsection{Infrared Properties}

The infrared spectrum of this material is shown in Figures $14 a$ and $14 b$. The $4000-1000 \mathrm{~cm}^{-1}(2.5-10 \mu \mathrm{m})$ spectrum, shown in Figure $14 b$, is dominated by strong absorptions at the lower frequencies. There is a clearly resolved aromatic $\mathrm{CH}$ stretch at about $3030 \mathrm{~cm}^{-1}$, with equally strong aromatic signatures at 1600 and $1200 \mathrm{~cm}^{-1}$. The aliphatic $\mathrm{CH}$ stretch between $3000-2800 \mathrm{~cm}^{-1}$ shows the presence of both $-\mathrm{CH}_{3}$ and $-\mathrm{CH}_{2}-$ groups. The doublet bands at 1460 and 1380 are assigned to the aliphatic $\mathrm{CH}$ deformations expected from the same groups that produce the aliphatic $\mathrm{CH}$ stretch complex between 3000-2800 $\mathrm{cm}^{-1}$. The comparable strength of the $\mathrm{CH}$ stretch to the $\mathrm{CH}$ deformation feature is a very strong indicator that the aliphatic component does not dominate this material, but rather is intimately mixed with other chemical subgroups. The absence of any absorption attributable to a carboxylic $\mathrm{OH}$ stretch in the $3 \mu \mathrm{m}$ region implies that the $1700 \mathrm{~cm}^{-1}(5.88 \mu \mathrm{m})$ carbonyl band is an indication of aldehydes and ketones. A significant aromatic component is implied by the sharp $1600 \mathrm{~cm}^{-1}$ component that likely arises from an aromatic $\mathrm{CC}$ stretch and the broad band between roughly 1300 and $1100 \mathrm{~cm}^{-1}$ which is characteristic of aromatic $\mathrm{CC}$ stretching and aromatic in-plane $\mathrm{CH}$ bending vibrations.

\subsubsection{Biological Material}

4.3.9.1. Material Preparation

A freeze-dried sample of Escherichia coli W1485 bacteria was placed in a methanol solution for dilution. A small amount of the mixture was then placed on a $\mathrm{KBr}$ window and, after warming to $40^{\circ} \mathrm{C}$ to remove the methanol, an infrared transmission spectrum was taken. Jonathan Trent and Hiromi Kagawa kindly provided the sample, and Bishun Khare generously took the spectrum.

4.3.9.2. Infrared Properties

The infrared spectrum of this material is shown in Figures $15 a$ and $15 b$. As the chemical nature of a biological system such as E. coli is extremely complex, a rich spectrum 

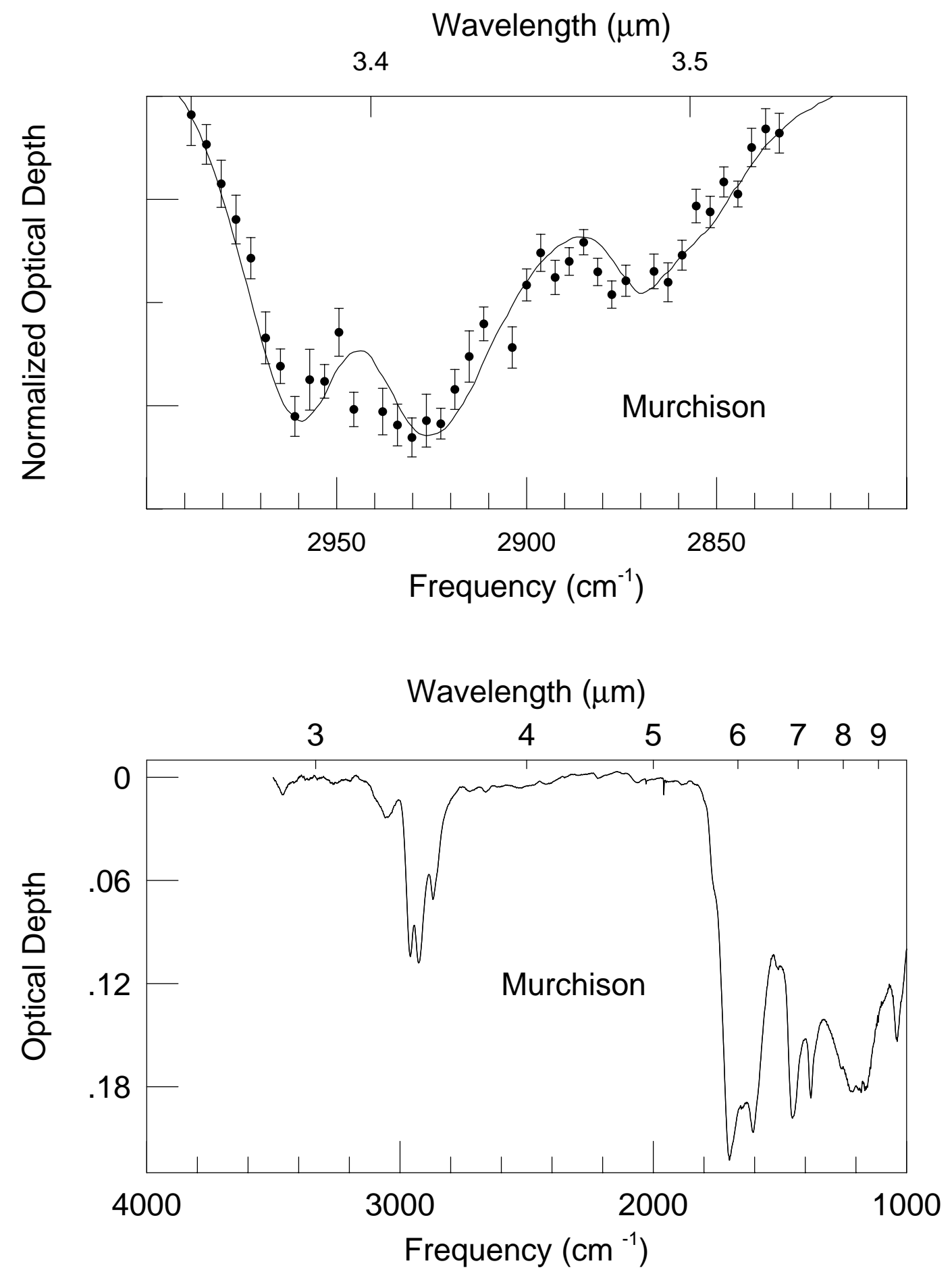

Fig. 14.- (a) Comparison of the $\mathrm{CH}$ stretch of the $600^{\circ} \mathrm{C}$ organic extract sublimate from the Murchison meteorite (DeVries et al. 1993, discussed in $\S 4.3 .8$ ) to the diffuse interstellar medium $3.4 \mu \mathrm{m}$ band (GC IRS 6E; Pendleton et al. 1994). (b) The $3600-1000 \mathrm{~cm}^{-1}(2.78-10 \mu \mathrm{m})$ spectrum of this Murchison extract. (Spectrum from DeVries et al. 1993 and sample preparation described in Kerridge et al. 1987).

is expected. Here, as above, we focus on the identification of the chemical subgroups responsible for particular absorption bands. Some shifting from the positions listed above in the simpler systems is expected as nearly any biological sample contains, among other things, acids, bases, and metals which can alter band positions and profiles.

The strong, broad absorption between roughly 3550 and perhaps $2400 \mathrm{~cm}^{-1}$ implies the presence of $\mathrm{OH}$ and $\mathrm{NH}$ bands as expected from carboxylic acid groups $(-\mathrm{COOH})$, alcohols $(-\mathrm{OH})$, amines $\left(-\mathrm{NH}_{2}\right)$, and possibly some trapped $\mathrm{H}_{2} \mathrm{O}$. The much deeper doublet near $1650 \mathrm{~cm}^{-1}$ and the deep $1540 \mathrm{~cm}^{-1}$ band are consistent with the carboxylic acid assignment and other carbonyl groups. The presence of several prominent features in this region indicates the importance of a variety of carbonyl types such as aldehydes, ketones, and amides (e.g., see Fig. 7). In general, all are abundant constituents of biosamples. The prominent absorptions centered at 1250 and $1060 \mathrm{~cm}^{-1}$ likely arise 

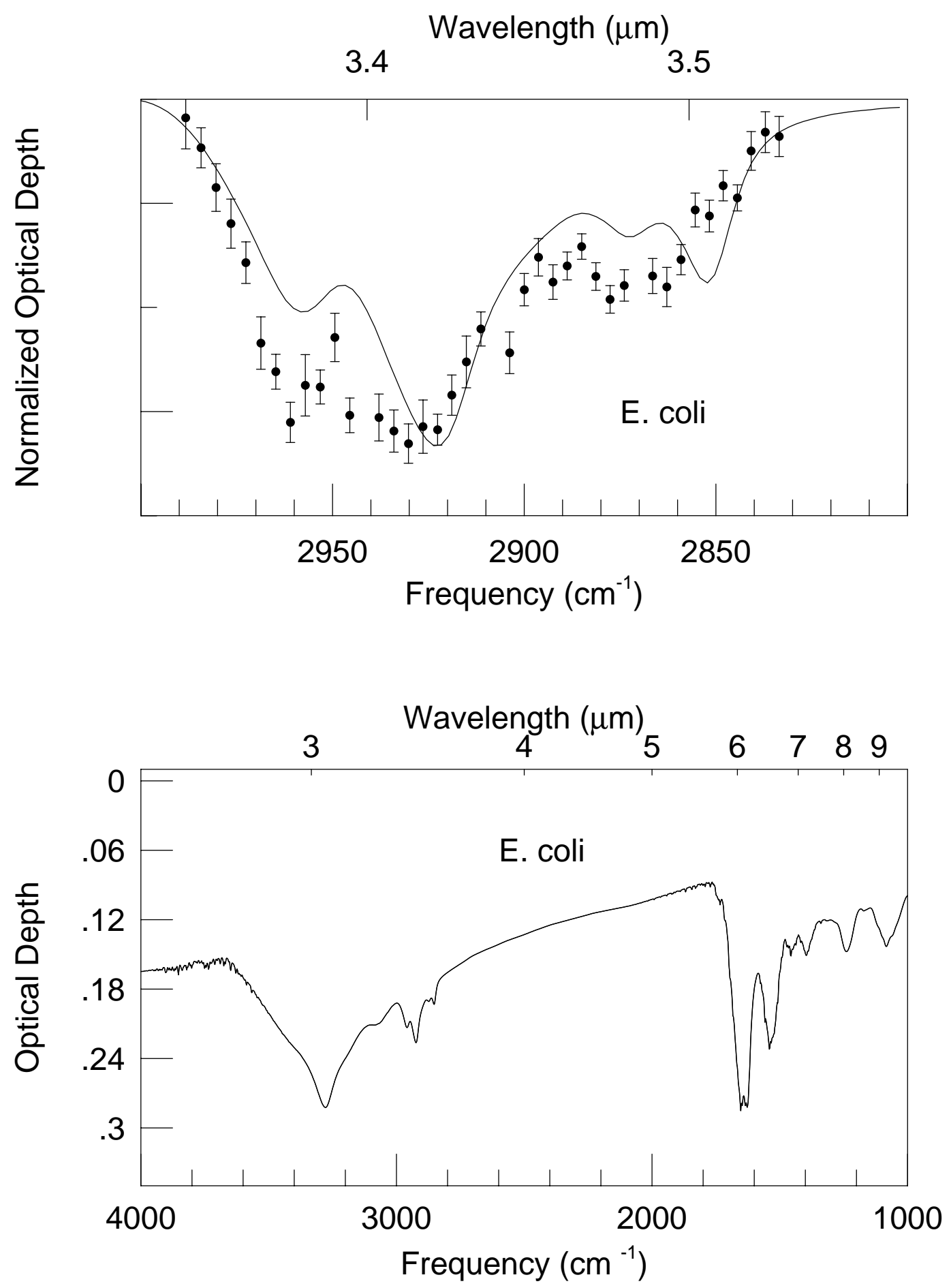

Fig. 15.-(a) Comparison of the $\mathrm{CH}$ stretch of a freeze-dried sample of Escherichia coli W1485 (courtesy of Jonathan Trent and Hiromi Kagawa; spectrum obtained by Bishun Khare) to the diffuse interstellar medium $3.4 \mu \mathrm{m}$ band (GC IRS 6E; Pendleton et al. 1994). (b) The 3600-1000 $\mathrm{cm}^{-1}(2.78-10$ $\mu \mathrm{m})$ spectrum of Escherichia coli W1485. Details of the sample preparation are given in $\S$ 4.3.9.

from stretching involving the singly bonded elements $\mathrm{C}, \mathrm{N}$, and $\mathrm{O} . \mathrm{OH}$ bending vibrations likely contribute to the absorption underlying the bands between about 1700 and $1000 \mathrm{~cm}^{-1}$.

The presence of aliphatic $-\mathrm{CH}_{3}$ and $-\mathrm{CH}_{2}-$ groups is shown by the structure in the band between 3000 and 2800 $\mathrm{cm}^{-1}$ (Fig. 15a). The $\mathrm{CH}$ deformation vibrations expected are clearly present at 1470 and $1400 \mathrm{~cm}^{-1}$. The strong relative intensities of the $\mathrm{CH}$ deformation bands to the $\mathrm{CH}$ stretching absorption complex centered near $2900 \mathrm{~cm}^{-1}$ are consistent with aliphatic material that is perturbed by nearby chemical subgroups. The $1400 \mathrm{~cm}^{-1}$ position (shifted from the more typical $1370 \mathrm{~cm}^{-1}$ ) is also consistent with this interpretation.

\subsubsection{Extragalactic Dust}

Figure 16 compares the DISM $3.4 \mu \mathrm{m}$ feature of our Galaxy with the organic signature of a distant galaxy, IRAS $08572+3915$. The dust observed in the distant galaxy $(z=0.05)$ has been corrected for redshift in the figure com- 

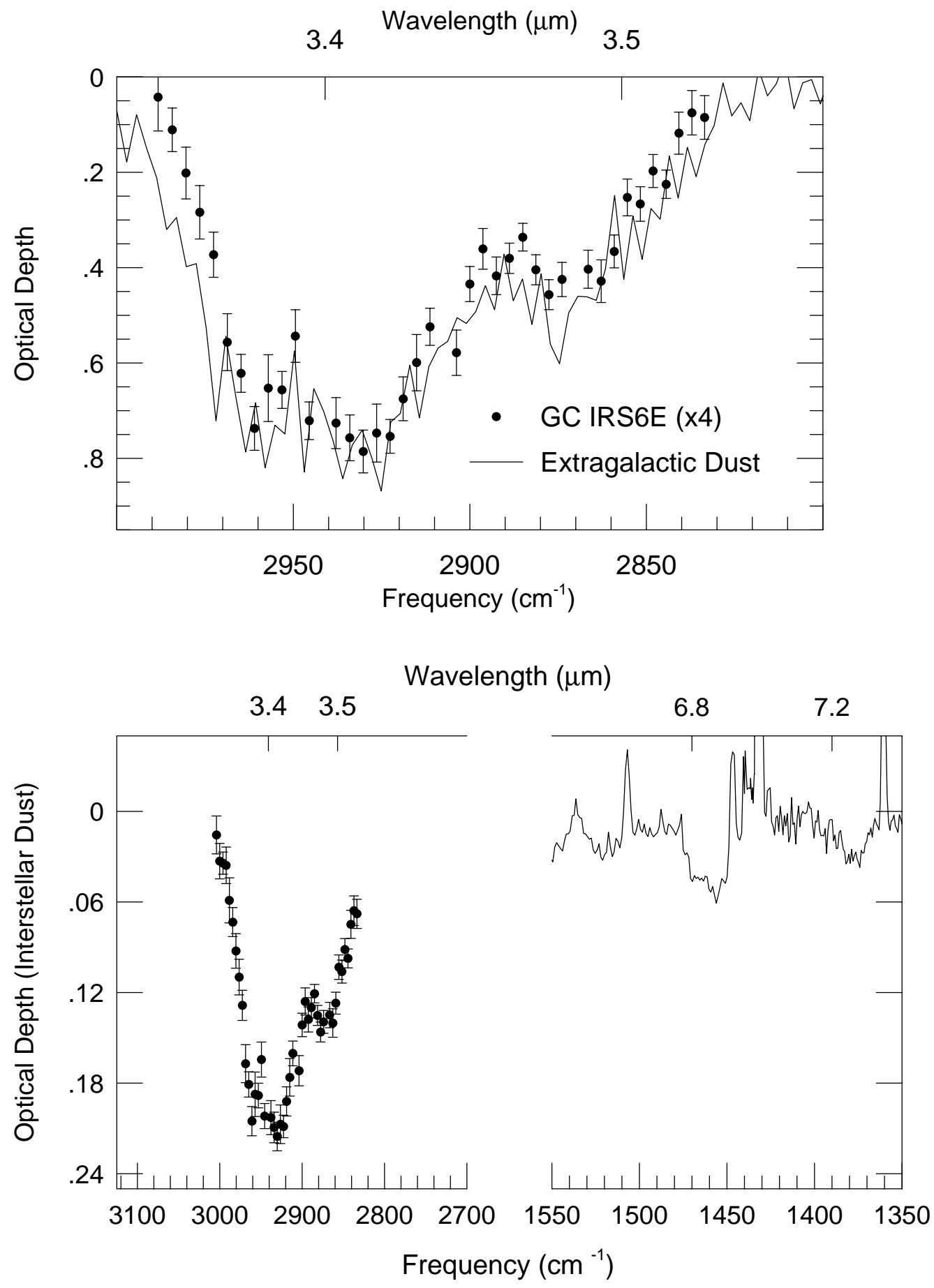

FIG. 16. - (a) Comparison of the galactic diffuse interstellar medium $3.4 \mu \mathrm{m}$ absorption features (GC IRS 6E; Pendleton et al. 1994; solid points) to that detected in the dust of the luminous galaxy IRAS 08572 + 3915 (Pendleton 1995, 1996a, 1996b; Wright et al. 1996; Imanishi \& Dudley 2000). The spectrum of IRAS $08572+3915$ (solid line) has been corrected for redshift. (b) Composite of the optical depth of the galactic diffuse interstellar medium $3.4 \mu \mathrm{m}$ and 5-8 $\mu$ m absorption features $\left(3100-2700 \mathrm{~cm}^{-1}\right.$, Pendleton et al. 1994, GC IRS 6E); (1550-1350 $\mathrm{cm}^{-1}$, Chiar et al. 2000, Sgr A*).

parison, and in so doing it is apparent that the subfeatures fall at the same positions as the aliphatic hydrocarbon absorption bands in our own Galaxy. The extragalactic dust signature is measured toward the bright nucleus of IRAS 08572 + 3915 (Pendleton 1996a, 1996b; Wright et al. 1996; Imanishi \& Dudley 2000). The striking match in the $3.4 \mu \mathrm{m}$ region suggests the longer wavelength region might also be similar to that of the diffuse dust in our own Galaxy. Unfortunately, moderate spectral resolution data for that important 5-10 $\mu \mathrm{m}$ region is not available for IRAS
$08572+3915$, and most of that spectral range cannot be observed from ground- based observatories. It is important to obtain $5-10 \mu \mathrm{m}$ spectra for this object, the strongest source of extragalactic aliphatic hydrocarbons known. The $3.4 \mu \mathrm{m}$ absorption band has been detected in a few other Seyfert galaxies, and observations in spectral windows available from the ground are being made in order to search for trends in dust properties and probe the geometries of these extragalactic environments (Wright et al. 1996; Imanishi 2000). 


\section{SPECTRAL CONSTRAINTS AND MATERIAL ASSESSMENT}

The observational data shown in Figure 3, and the power of infrared spectroscopy, allow significant constraints to be placed on candidate materials proposed as relevant to the organic refractory material in the DISM. The keystone that supports this analysis is the firm detection of the aliphatic $\mathrm{CH}$ stretch feature along the line of sight to Cyg OB2 No. 12 (see Fig. 4 for profile comparison with galactic center line of sight toward GC IRS 6E). The presence of the aliphatic absorption near $3.4 \mu \mathrm{m}$ implies the presence of corresponding features in the 5-9 $\mu \mathrm{m}$ region. By placing upper limits on the interstellar "features," constraints can be applied to candidate materials which offer a good fit to the $3.4 \mu \mathrm{m}$ band, but which exhibit spectral features that are inconsistent with the 5-9 $\mu \mathrm{m}$ upper limits of the interstellar data.

The four spectral criteria outlined here enable us to assess the merit of the various candidate materials. Based on the interstellar data, these criteria are (i) the $2900 \mathrm{~cm}^{-1}(3.4 \mu \mathrm{m})$ aliphatic $\mathrm{CH}$ stretch band profile and subpeak positions, (ii) the ratio of the optical depth of the aliphatic $\mathrm{CH}$ stretch to the optical depth of the $\mathrm{OH}$ stretch near $3200 \mathrm{~cm}^{-1}(3.1$ $\mu \mathrm{m})$, (iii) the ratio of the optical depth of the aliphatic $\mathrm{CH}$ stretch to the optical depth of the carbonyl band near 1700 $\mathrm{cm}^{-1}(5.9 \mu \mathrm{m})$, and (iv) the ratio of the optical depth of the aliphatic $\mathrm{CH}$ stretch feature to the optical depth of the $\mathrm{CH}$ deformation modes near $1470 \mathrm{~cm}^{-1}(6.8 \mu \mathrm{m})$ and 1370 $\mathrm{cm}^{-1}(7.25 \mu \mathrm{m})$. These criteria will now be discussed in turn, and the extent to which the various candidate materials satisfy them is indicated in Table 2 by a plus $(+)$ or minus (-) sign.

(i) The $2900 \mathrm{~cm}^{-1}(3.4 \mu \mathrm{m})$ aliphatic $\mathrm{CH}$ stretch band profile.-The degree to which the band profile has been applied as a discriminant to the various candidate materials has evolved considerably from the early 1980's when it was simply referred to as the $3.4 \mu \mathrm{m}$ band and any material absorbing close to this frequency was considered a possible "fit." The quality of the interstellar data significantly improved in the early 1990's, providing the necessary higher spectral resolution and high signal-to-noise ratio required to discuss meaningful "fits." Careful analysis of the detailed interstellar band profile reveals much about the type of hydrocarbon implied, offers some understanding of the general properties of the organic refractory material, and gives a firm lower limit to the amount of available $\mathrm{C}$ tied up in this material. These conclusions, summarized below in $\S 6$, are taken from Sandford et al. (1991) and Pendleton et al. (1994).

Figures 5, 8, 10, 12, 14, and 15 show detailed comparisons between the interstellar $\mathrm{CH}$ stretch feature and the bands associated with each of the materials considered here. A plus $(+)$ or minus $(-)$ sign is assigned for this criterion as follows. To warrant a plus, the candidate feature had to closely reproduce the three subpeak positions in the DISM spectrum at 2955,2925 , and $2870 \mathrm{~cm}^{-1}$ as well as their relative intensities and widths. For example, the comparison shown in Figures $5 a$ and $5 b$ do not meet these criteria and are assigned a minus in the table. On the other hand,

TABLE 2

An Assessment of Spectral Comparisons of Laboratory Materials to the Interstellar Observations Showing that only Carbonaceous Materials Meet the Observational Criteria

\begin{tabular}{|c|c|c|c|c|}
\hline \multirow[b]{2}{*}{ MATERIAL } & \multicolumn{4}{|c|}{ CRITERIA } \\
\hline & $\underset{3.4 \mu \mathrm{m} \text { Profile }}{\mathrm{CH}_{\mathrm{S}}}$ & $\begin{array}{c}\mathrm{CH}_{\mathrm{S}} / \mathrm{OH}_{\mathrm{S}} \\
\tau_{3.4} / \tau_{3.0}\end{array}$ & $\begin{array}{c}\mathrm{CH}_{\mathrm{S}} / \mathrm{CO}_{\mathrm{S}} \\
\tau_{3.4} / \tau_{5.9}\end{array}$ & $\begin{array}{c}\mathrm{CH}_{\mathrm{S}} / \mathrm{CH}_{\mathrm{B}} \\
\tau_{3.4} / \tau_{6.8}\end{array}$ \\
\hline \multicolumn{5}{|c|}{ Ice Residue: Produced by UV Irradiation } \\
\hline Ice residue 4.1.1 (Fig. 6a) & - & $?$ & + & + \\
\hline Ice residue 4.1 .2 (Fig. $6 b$ ) & - & $?$ & - & $?$ \\
\hline Ice residue 4.1 .3 (Fig. $6 c$ ) & + & - & - & - \\
\hline \multicolumn{5}{|c|}{ Ice Residue: Produced by Energetic Particles } \\
\hline Ice residue 4.2.1 (Fig. 9a) & - & + & + & + \\
\hline Ice residue 4.2 .2 (Fig. $9 b$ ) & + & - & - & + \\
\hline Ice residue 4.2 .3 (Fig. $9 c$ ) & + & - & $?$ & + \\
\hline \multicolumn{5}{|c|}{ Carbonaceous Materials: Produced from Carbon Rods and Plasmas } \\
\hline Heated carbon rod 4.3 .1 (Fig. 11a) & + & + & + & + \\
\hline Laser ablated carbon rod + hydrogenation 4.3 .2 (Fig. $11 b)$ & + & + & + & + \\
\hline Aromatic electric discharge 4.3 .3 (Fig. 11c) & $(+)$ & + & + & + \\
\hline Methane electric discharge 4.3.4 (Fig. 11d) & + & + & + & + \\
\hline Methane microwave discharge 4.3 .5 (Fig. 13a) & - & + & + & + \\
\hline Methane microwave discharge-heated 4.3.6 (Fig. 13b) & $?$ & + & $?$ & - \\
\hline Laser ablated graphite in $\mathrm{H}_{2}$ atmosphere 4.3 .7 (Fig. 13c) & $(+)$ & + & + & + \\
\hline \multicolumn{5}{|c|}{ Murchison Meteorite Extract } \\
\hline Murchison carbon extract 4.4 (Fig. 14) & + & + & - & $?$ \\
\hline \multicolumn{5}{|c|}{ Bacterium } \\
\hline Freeze-dried E. coli 4.5 (Fig. 15) & - & - & - & + \\
\hline
\end{tabular}

Notes.-A plus sign $(+)$ means the material satisfies the criterion, a minus sign $(-)$ means the material fails, and a interrogative sign (?) means it is not possible to draw a conclusion. Subscripts "S" and "B" indicate stretch or bend, respectively. 
those shown in Figures $8 b, 8 c, 10 a, 10 b, 10 c$, and $12 c$ warrant a plus. An interrogative sign (?) indicates insufficient information to apply this test.

(ii) Ratio of the optical depth of the aliphatic $\mathrm{CH}$ stretch feature to the optical depth of the $\mathrm{OH}$ stretch peak near 3200 $\mathrm{cm}^{-1}(3.1 \mu \mathrm{m})$.- Figure $3 a$ shows that, within the limit of the error bars, there is no evidence for $\mathrm{OH}$ absorption near $3200 \mathrm{~cm}^{-1}$ in the spectrum of Cyg OB2 12. Deriving an upper limit to the optical depth $(\tau)$ at this position based on roughly half of the error bar values, as listed in Table 1, we deduce $\tau_{3200 \mathrm{~cm}^{-1}}<0.02$. This compares with the $\tau_{2900 \mathrm{~cm}^{-1}}=0.04$ for the aliphatic CH stretch band toward this object (Sandford et al. 1991; Pendleton et al. 1994; Whittet et al. 1997). Given that the intrinsic strength of the $\mathrm{OH}$ feature per $\mathrm{OH}$ group is typically 10-20 times the intrinsic strength of the $\mathrm{CH}$ stretch band per methyl or methylene group (e.g., d'Hendecourt \& Allamandola 1986), the amount of oxygen tied up in the organic refractory component of the DISM as hydrogen bonded $\mathrm{OH}$ is negligible and represents at most only a few percent of the carbon.

Figures $6,9,11,13,14$, and 15 show the entire mid-IR spectrum of each of the materials considered here. A plus $(+)$ or minus $(-)$ sign was assigned for this criterion as follows. To warrant a plus the $\tau$ of the aliphatic $\mathrm{CH}$ stretch band (not including a contribution from an underlying $\mathrm{OH}$ stretch band, if present) in the candidate material had to be twice as strong as the $\tau$ of the $\mathrm{OH}$ stretch peaking near 3250 $\mathrm{cm}^{-1}$. As an example, the O.D. of the aliphatic $\mathrm{CH}$ stretch shown in Figure $9 b$ is about 0.15 , while the $\tau$ of the $\mathrm{OH}$ stretch which peaks near $3300 \mathrm{~cm}^{-1}$ in this material is slightly larger. Thus, the $\mathrm{CH}$ stretch to $\mathrm{OH}$ stretch band optical depth ratio is less than 1 , and this material is assigned a minus in the table. Similarly, as the $\tau$ of the $\mathrm{OH}$ stretch band near $3300 \mathrm{~cm}^{-1}$ is about 2.5 times larger than the optical depth of the aliphatic $\mathrm{CH}$ stretch in the spectrum shown in Figure $6 c$, this too is assigned a minus in Table 2. Conversely, the spectra of the materials shown in Figures $11 a, 11 b, 11 c$, and $11 d$ satisfy this criterion and are assigned a plus in the table.

(iii) Ratio of the optical depth of the aliphatic $\mathrm{CH}$ stretch feature to the optical depth of the carbonyl band near 1700 $\mathrm{cm}^{-1}(5.9 \mu \mathrm{m})$. - Figure 3 shows that, within the limit of the error bars, there is no evidence for carbonyl absorption near $1700 \mathrm{~cm}^{-1}$ in the spectrum of Cyg ОВ2 No. 12. Unfortunately, the error bars in this portion of the spectrum are larger than those at the higher frequencies, reducing the stringency of this constraint. Deriving an upper limit to the optical depth at this position based on roughly half of the error bar values, as listed in Table 1, we deduce $\tau_{1700 \mathrm{~cm}^{-1}}<0.06$. Again, this must be compared with the $\tau_{2900 \mathrm{~cm}^{-1}}=0.04$ of the aliphatic $\mathrm{CH}$ stretch band toward this object. Given that the intrinsic strength of the carbonyl feature is typically at least twice as strong as the intrinsic strength of the $\mathrm{CH}$ stretch band per methyl or methylene group and is as much as 10 times stronger in many cases (e.g., Wexler 1967; d'Hendecourt \& Allamandola 1986), the amount of oxygen and carbon tied up as carbonyl groups in the organic refractory component of the DISM material is less than half that of the aliphatic carbon in $\mathrm{CH}$ and is likely much less.

Figures $6,9,11,13,14$, and 15 show the entire mid-IR spectrum of each of the materials considered here. A plus $(+)$ or minus (-) sign was assigned for this criterion as follows. To warrant a plus the optical depth of the aliphatic $\mathrm{CH}$ stretch band (not including a contribution from an underlying $\mathrm{OH}$ stretch if present) in the candidate material had to be comparable to or stronger than the optical depth of the carbonyl band peaking near $1700 \mathrm{~cm}^{-1}$. As an example, the O.D. of the aliphatic $\mathrm{CH}$ stretch shown in Figure $6 a$ is about 0.05 , while the optical depth of the carbonyl peak near $1700 \mathrm{~cm}^{-1}$ is about 0.06 . Thus, the $\mathrm{CH}$ stretch to carbonyl $\mathrm{CO}$ stretch band optical depth ratio is less than 1, and this material is assigned a minus in the table. Similarly, as the optical depths of the aliphatic $\mathrm{CH}$ stretch in the spectra shown in Figures $6 c$ and $9 b$ are much less than half the optical depth of the carbonyl band near $1700 \mathrm{~cm}^{-1}$, these too are assigned a minus in the table. Conversely, all of the spectra of the materials shown in Figure 11 satisfy this criterion and are assigned a plus in the table.

(iv) Ratio of the optical depth of the aliphatic $\mathrm{CH}$ stretch feature to the aliphatic $\mathrm{CH}$ deformation modes near 1470 $\mathrm{cm}^{-1}(6.8 \mu \mathrm{m})$. - This is the weakest constraint allowable by the spectral quality of the data in Figure $3 c$. As discussed above, the subpeak positions of the $\mathrm{CH}$ stretch band simply indicate that there are $-\mathrm{CH}_{3}$ and $-\mathrm{CH}_{2}-$ groups present in the material and the profile gives insight into the nature of the material these groups are attached to and which perturbs them. The entire mid-infrared spectrum, however, gives deeper insight into the overall nature of the hydrocarbon than does the $2900 \mathrm{~cm}^{-1}$ band alone. Materials which are primarily pure alkanes absorb much more strongly in the $\mathrm{CH}$ stretch band than in the $\mathrm{CH}$ deformation band. For example, the absorbance ratio (equivalent to optical depth ratio) of the $\mathrm{CH}$ stretch to the $\mathrm{CH}$ deformation bands in solid pentane $\left(\mathrm{CH}_{3}\left(\mathrm{CH}_{2}\right)_{3} \mathrm{CH}_{3}\right)$ and hexane $\left(\mathrm{CH}_{3}\right.$ $\left.\left(\mathrm{CH}_{2}\right)_{4} \mathrm{CH}_{3}\right)$ are 4.6 and 5.1, respectively. Similarly, the ratio is greater than 10 in octanol $\left(\mathrm{CH}_{3}\left(\mathrm{CH}_{2}\right)_{7} \mathrm{OH}\right)$, an alcohol with a large alkane chain. For molecules which have comparable mixtures of alkane groups to other types of chemical subgroups, such as acetonitrile $\left(\mathrm{CH}_{3} \mathrm{CN}\right)$ or ethylacetate $\mathrm{CH}_{3}\left(\mathrm{CO}_{2}\right) \mathrm{CH}_{2} \mathrm{CH}_{3}$, the ratios are typically close to or less than 1 . In these two examples, the ratios are 0.7 and 0.4 , respectively (e.g., d'Hendecourt \& Allamandola 1986). Thus, the lower the ratio of the $2900 \mathrm{~cm}^{-1}$ optical depth to the $1470 \mathrm{~cm}^{-1}$ optical depth, the lower the pure alkane character of the interstellar material. Low ratios indicate other types of chemical subgroups are present as well, in close proximity to the methyl $\left(-\mathrm{CH}_{3}\right)$ and methylene $\left(-\mathrm{CH}_{2}-\right)$ groups.

The optical depth of the $2900 \mathrm{~cm}^{-1}$ aliphatic $\mathrm{CH}$ stretch band toward Cyg OB2 No. 12 is $0.04 \pm 0.002$ (Sandford et al. 1991; Pendleton et al. 1994; Whittet et al. 1997). Analysis of the lower frequency portion of the spectrum as described in $\S 3$ shows that the maximum optical depth possible at about $1470 \mathrm{~cm}^{-1}$, the aliphatic $\mathrm{CH}$ deformation position, is 0.04 (Table 1). Thus, within the limits allowed by the interstellar spectrum currently available, the ratio of the aliphatic $\mathrm{CH}$ stretch to deformation bands toward Cyg OB2 No. 12 is $\geq 1$. This rules out materials in which small aliphatic groups $(n<2-3)$ are dominated by other chemical subgroups, because in that case the ratio of the stretch to deformation bands will not be greater than or comparable to one. The recent detection of bands attributable to the aliphatic $\mathrm{CH}$ deformation modes along the line of sight to the galactic center (Chiar et al. 2000), shown here in Figure $16 b$, constrains the situation further. Toward the galactic 
center, the optical depth of the $\mathrm{CH}$ stretch is roughly 0.2 and the deformation bands at 1460 and $1380 \mathrm{~cm}^{-1}(6.85$ and $7.35 \mu \mathrm{m}$ ) have optical depths of 0.04 and 0.02 , respectively (Chiar et al. 2000). As discussed above, this ratio $(\sim 5)$ is consistent with maximum alkane chain lengths of about 4 interspersed most likely with aromatic entities. This suggests, therefore, that DISM organic refractory material along this line of sight is characterized by roughly equivalent, intimate mixtures of methyl and methylene groups with other chemical subgroups. Long alkane chains $\mathrm{H}_{3} \mathrm{C}-\left(\mathrm{CH}_{2}\right)_{n}-$ with $n$ much greater than 4 or 5 are not major constituents of this material.

Figures $6,9,11,13,14$, and 15 show the entire mid-IR spectrum of each of the materials considered here. A plus $(+)$ or minus (-) sign in Table 2 was assigned for this criterion as follows. To warrant a plus the optical depth of the aliphatic $\mathrm{CH}$ stretch band (not including a contribution from an underlying $\mathrm{OH}$ stretch if present) in the candidate material had to be greater than or equal to the optical depth of the deformation band near $1480 \mathrm{~cm}^{-1}$. For example, the O.D. of the aliphatic $\mathrm{CH}$ stretch shown in shown in Figure $9 a$ is between 0.008 and 0.009 , while the optical depth in the deformation region near $1480 \mathrm{~cm}^{-1}$ is between 0.002 and 0.004. Thus, the $\mathrm{CH}$ stretch-to-deformation band $\tau$ ratio is greater than 1 and this material is assigned a plus in the table. Similarly, the optical depth of the aliphatic $\mathrm{CH}$ stretch in the spectrum shown in Figure $9 b$ is about 0.15 (disregarding the contribution from the underlying $\mathrm{OH}$ band), while the optical depth near $1480 \mathrm{~cm}^{-1}$ is between 0.1 and 0.2 . As these are comparable within the errors of the interstellar spectra, this is also assigned a plus.

\section{THE NATURE OF THE DIFFUSE INTERSTELLAR ORGANIC REFRACTORY MATERIAL AND ASTROPHYSICAL IMPLICATIONS}

\subsection{The Organic Refractory Component of the DISM}

Summarizing the previous two sections, Table 2 shows that the organic refractory component of the diffuse interstellar medium is largely hydrocarbon in nature. Containing little $\mathrm{O}$ or $\mathrm{N}$, the carbon is distributed between the aliphatic and aromatic forms. The interstellar spectrum toward CYG OB2 No. 12 shows that the diffuse interstellar material does not contain much oxygen in the alcohol, acid, or carbonyl forms. These groups can be, at most, only a few percent of the carbon. In addition, the lack of detectable absorption in the $2200-2000 \mathrm{~cm}^{-1}(4.55-5 \mu \mathrm{m})$ nitrile $(-\mathrm{C} \equiv \mathrm{N})$ region shows this group is not an important component of the diffuse interstellar organic refractory material. The $2900 \mathrm{~cm}^{-1}(3.4 \mu \mathrm{m})$ band substructure and profile, together with the optical depth ratio of the $\mathrm{CH}$ stretch band to the $\mathrm{CH}$ deformation band, show that the organic refractory material in the DISM contains $-\mathrm{CH}_{3}$ and $-\mathrm{CH}_{2}-$ groups whose individual chain lengths are not likely to be much longer than 4 or 5 , and that these short aliphatic chains are attached to electronegative chemical groups. Table 2 reveals that of all the candidates considered, the plasma processed carbonaceous materials satisfy all the spectroscopic criteria.

Together, the profile of the $3.4 \mu \mathrm{m}$ feature, the $\mathrm{CH}$ stretch-to-deformation mode optical depth ratio, and the absence of evidence for other types of chemical subgroups, provides indirect evidence that aromatic, $\mathrm{sp}^{2}$ hybridized
TABLE 3

\begin{tabular}{|c|c|}
\hline $\begin{array}{c}\text { The Distribution of CA } \\
\text { IN THE DifFuSE INTERSTE } \\
\text { Medium DUST SHOWN } \\
\text { FIGURE } 17\end{array}$ & \\
\hline Aromatic & \\
\hline $\begin{array}{c}\mathrm{C} \text { atoms } . . . \ldots \ldots \ldots \ldots \ldots \\
\mathrm{C}-\mathrm{H} \text { bonds } . . . \ldots \ldots \ldots \ldots . . .\end{array}$ & $\begin{array}{l}400 \\
140\end{array}$ \\
\hline Aliphatic & \\
\hline C atoms............. & 70 \\
\hline $\mathrm{C}-\mathrm{H}$ bonds...$\ldots \ldots \ldots \ldots$ & 150 \\
\hline Methyl $-\mathrm{CH}_{3} \ldots \ldots \ldots \ldots$ & 40 \\
\hline Methylene $-\mathrm{CH}_{2}-\ldots \ldots$ & 100 \\
\hline Tertiary $\mathrm{CH} \ldots \ldots \ldots \ldots \ldots$ & 5 \\
\hline
\end{tabular}

carbon domains (aromatics) are an important component of the interstellar material. Due to the intrinsic weakness of the CC stretch modes, they could be present in comparable amounts to the aliphatics, thereby influencing the $\mathrm{CH}$ stretch-to-CH deformation optical depth ratio, while not adding other significant features to the spectrum. The detection of the aromatic band near $6 \mu \mathrm{m}$ detected along several lines of sight through the DISM (Schutte et al. 1998; Keane et al. 2001) supports this view of the organic component of DISM dust. It should be noted that the observed $6 \mu \mathrm{m}$ feature has been shown to be circumstellar rather than interstellar in origin (Chiar \& Tielens 2001). An example of the type of chemical stucture which satisfies all of these constraints is shown in Figure 17, and the distribution of carbon is given in Table 3. A rotating, three-dimensional representation can be found on the World Wide Web. ${ }^{2}$ Last, diamond-like carbon domains may also be present; however, the IR spectra currently available cannot address this possibility.

\subsection{Astrophysical Implications}

These hydrocarbons appear to be made in the outflow of evolved carbon stars (Lequeux \& Jourdain de Muizon 1990; Chiar et al. 1998). Production of hydrocarbons in the outflow of stars and the subsequent balance between hydrogenation and UV-dehydrogenation may follow the path suggested by Mennella et al. (1999) and Mennella et al. (2001). Likewise, the thermal evolution of coal-like analogs of interstellar dust exhibits similar spectral modification (Papoular et al. 1996 and Guillois et al. 1996). That the aliphatic signature is present along a dozen different sight lines through the diffuse ISM (Sandford et al. 1991; Pendleton et al. 1994) suggests the production dominates destruction in many regions of the diffuse ISM. However, as the aliphatic signature has not been observed in dense cloud environments thus far, some mechanism must be active at the diffuse/dense cloud interface which reduces the aliphatic component.

Given the short cycling time of dust between the diffuse and dense clouds (McKee 1989), and the widespread distribution of the organic refractory material which carries the aliphatic signature throughout the diffuse ISM, the absence of the $3.4 \mu \mathrm{m}$ signature in dense cloud spectra has been surprising (Allamandola et al. 1992). While this behavior is puzzling and no detailed explanation has been put

\footnotetext{
${ }^{2}$ See http://www.astrochem.org/ApJS2002/Fig17.html.
} 
No. 1, 2002

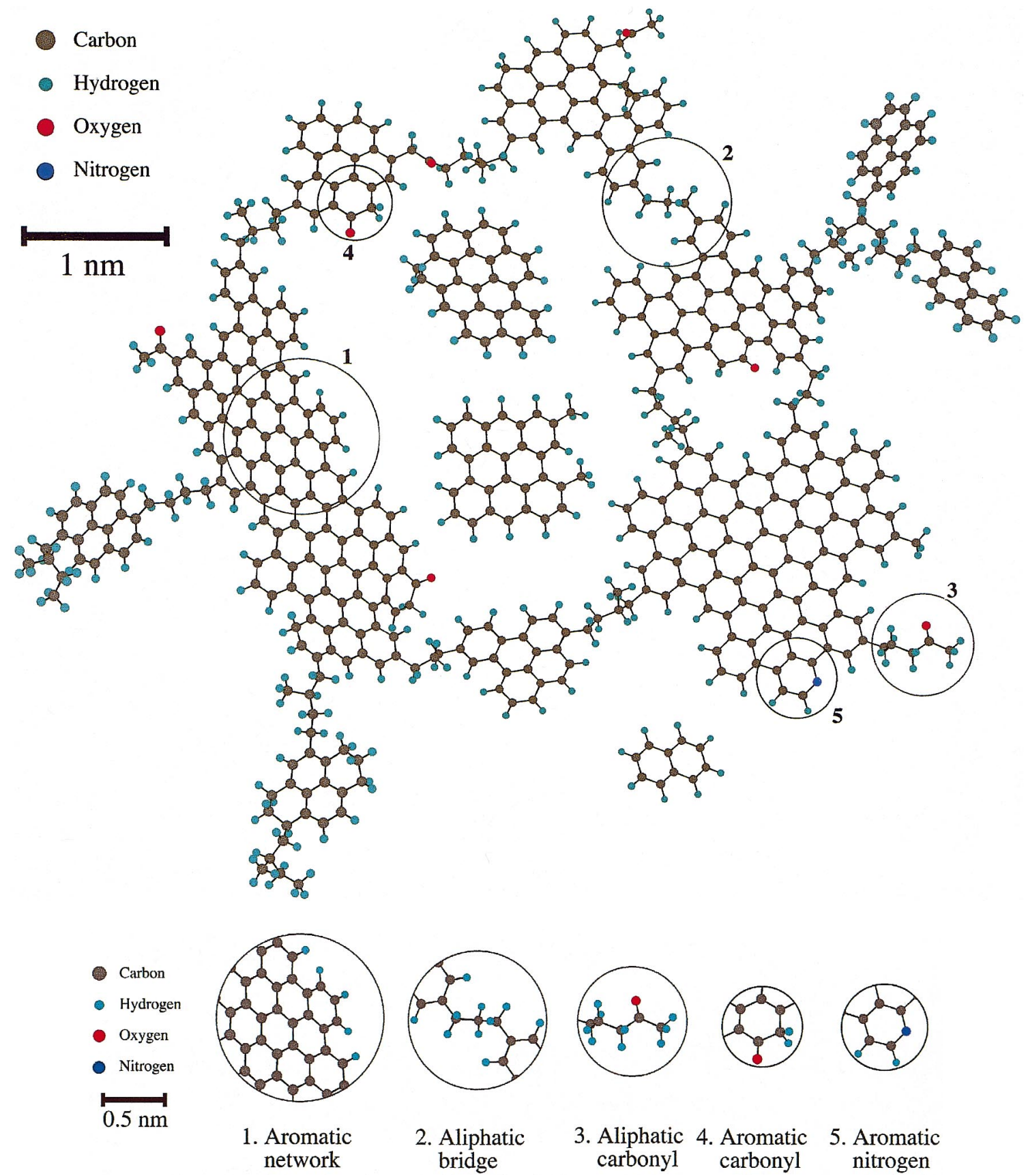

FIG. 17. - (a) The basic structural and molecular character of carbonaceous, interstellar dust in the diffuse interstellar medium. The molecular details have been deduced from the spectroscopic constraints as discussed in $\$ \S 5$ and 6 . The specific geometries of the aromatic plates and aliphatic components simply represent what is likely. The structure is somewhat splayed out to reveal the molecular structural details; we envision the actual structure somewhat more closed in. For the interconnected species (not the free-floating entities), the relative numbers of aromatic and aliphatic carbon-hydrogen bonds, as well as their subclassification within type, are all consistent with the observed spectrum described here. The latter includes the aliphatic $-\mathrm{CH}_{3}$ to $-\mathrm{CH}_{2}-$ ratio, and the relative numbers of aromatic solo, duo, trio, and quartet hydrogens deduced from the interstellar IR emission bands as described in Hony et al. (2001). The approximate volume of this fragment is on the order of $10^{-19} \mathrm{~cm}^{3}$. Thus, a typical $0.1 \mu \mathrm{m}$ DISM carbonaceous dust grain would contain approximately $10^{4}$ of these fragments. The encircled regions are expanded showing the different structural units. The carbon atom distribution of this fragment is summarized in Table 3 .

forth, Mennella et al. (1999) have suggested that the aliphatic component makes its way into the dense cloud, where it is destroyed by irradiation within the cloud. Once deep in the cloud, ice layers may further inhibit the formation of new $\mathrm{CH}$ bonds (Menella et al. 1999). Interestingly, these hydrocarbon grains may also be important in the conversion of $\mathrm{H}$ to $\mathrm{H}_{2}$ (Pirronello et al. 1999). A thorough discussion of the life cycle of grains can be found in Jones, Tielens, \& Hollenbach (1996) and Tielens (1998).

\subsection{Other Carbonaceous Materials}

Comparison of the DISM 3.4 um feature to that of the Murchison meteorite extract (Fig. 14a) indicates a remarkable similarity, and it has been suggested that an unaltered 
interstellar component might survive incorporation into the meteorite parent body (Ehrenfreund et al. 1991; Pendleton et al. 1994). Indeed, the inclusion of circumstellar grains, the deuterium content of the organics, and isotopic anomalies within the Murchison meteorite all indicate the preservation of material from the ISM (Kerridge et al. 1987; Ming \& Anders 1988; Lewis et al. 1994; Anders \& Zinner 1993; Cronin et al. 1988; Bernatowicz et al. 1996). However, in the 5-10 $\mu \mathrm{m}$ region, there are many strong features arising from materials that are definitely not present in the DISM observations. If the aliphatic material in the meteorite is unaltered interstellar material, then subsequent processing in either the solar nebula or on the parent body created new molecules which complicate the 5-10 $\mu \mathrm{m}$ spectrum.

Whether or not the aliphatic signature in the meteorite is interstellar, the implications for the evolution of interstellar grains are noteworthy. If the component in the meteorite is interstellar, then it must have been present in the dense cloud from which the Solar System formed, yet to date the $3.4 \mu \mathrm{m}$ feature has not been detected in dense cloud dust. If the DISM aliphatics are also present in dense molecular clouds, then newly forming planetary systems have access to these basic building blocks of biogenic material. On the other hand, if the aliphatics are truly absent in the dense clouds, then any explanation, such as the destruction scenario given above, must be made rigorous to understand the absence in the dense cloud in view of the short timescale in which grain material is thought to cycle between the diffuse and dense cloud regimes.

Comparisons of the galactic $3.4 \mu \mathrm{m}$ feature to E. coli bacteria have been presented in the literature in support of the notion that bacteria exist in space (e.g., Hoyle et al. 1982; Hoyle \& Wickramasinghe 1993, and others). The comparison presented here shows that the higher resolution interstellar data do not match the E. coli subfeatures well in the $3.4 \mu \mathrm{m}$ region, nor do the strong features in the $5-10 \mu \mathrm{m}$ region of the bacterium spectrum satisfy the upper limit criteria for possible features in the diffuse dust. Unlike the case of the meteorite, it is difficult to argue that additional components cause the difference between the bacterium and the diffuse ISM. If the $3.4 \mu \mathrm{m}$ feature of the bacterium were indeed responsible for the interstellar $3.4 \mu \mathrm{m} \mathrm{CH}$ stretch band, then the long-wavelength features must also be present. The absence of absorption attributable to any $\mathrm{O}-\mathrm{H}$ or carbonyl stretch toward Cyg OB2 No. 12 indicates that the dust in the diffuse medium does not contain significant amounts of $\mathrm{O}$ as $\mathrm{H}$-bonded $\mathrm{O}-\mathrm{H}$, either as part of the hydrocarbon molecules which produce the $\mathrm{C}-\mathrm{H}$ feature, or as $\mathrm{H}_{2} \mathrm{O}$ molecules trapped in an $\mathrm{H}$-bonded material or in carbonyl groups. Therefore, as the spectra of the ISM and the bacterium across the $2.5-10 \mu \mathrm{m}$ wavelength region do not match, we conclude there is no spectral evidence for bacteria in space. This is consistent with the earlier conclusions of Davies et al. (1984), Duley (1984), and Whittet (1984), among others, despite continued efforts to promote this idea (Hoyle \& Wickramasinghe 1991, 1993, 2000).

Comparisons of the galactic $3.4 \mu \mathrm{m}$ feature to the extragalactic observations of IRAS $08572+3915$ (Fig. 15a) indicate a remarkable match in spectral profile of the subfeatures due to the asymmetric $\mathrm{CH}_{3}$, symmetric $\mathrm{CH}_{2}$, and symmetric $\mathrm{CH}_{3}$ stretches $\left(2955,2925\right.$, and $2870 \mathrm{~cm}^{-1}$, respectively). Whether or not the 5-8 $\mu \mathrm{m}$ regions are similar is currently unknown as those data cannot be obtained from ground-based telescopes. A thorough review of all the organic material (dust and molecules) in the interstellar medium can be found in Ehrenfreund \& Charnley (2000).

\section{CONCLUSIONS}

Analysis of the $4000-1000(2.5-10 \mu \mathrm{m})$ region of the diffuse interstellar dust spectrum as seen toward Cyg OB2 No. 12, and the spectral comparison with various materials proposed as analogs of the refractory material in the diffuse interstellar medium, stress the critical importance of this frequency range in furthering our knowledge of the organic component of the interstellar medium. We conclude that the organic refractory component of the diffuse interstellar medium appears to be largely hydrocarbon in nature, possessing little $\mathrm{N}$ or $\mathrm{O}$, with carbon distributed between the aromatic and aliphatic forms.

Spectral analysis of the DISM over the $2.5-10 \mu \mathrm{m}$ wavelength range allows us to place significant constraints on candidate materials proposed as relevant to the interstellar organic refractory component. Of the 15 materials considered here (including 13 laboratory samples, an extract from the Murchison meteorite, and the spectrum of E. coli bacteria), only five satisfy all of the observational constraints. The spectra of candidate materials were evaluated using four spectral characteristics: (i) comparisons of the profile and subpeak positions of the $2940 \mathrm{~cm}^{-1}(3.4 \mu \mathrm{m})$ aliphatic $\mathrm{CH}$ stretch band, (ii) the ratio of the optical depth $(\tau)$ of the aliphatic $\mathrm{CH}$ stretch to the $\tau$ of the $\mathrm{OH}$ stretch near $3200 \mathrm{~cm}^{-1}(3.1 \mu \mathrm{m})$, (iii) the ratio of the $\tau$ of the aliphatic $\mathrm{CH}$ stretch to the $\tau$ of the carbonyl band near 1700 $\mathrm{cm}^{-1}(5.9 \mu \mathrm{m})$, and (iv) the ratio of the $\tau$ of the aliphatic $\mathrm{CH}$ stretch feature to the $\tau$ of the $\mathrm{CH}$ deformation modes near $1470 \mathrm{~cm}^{-1}(6.8 \mu \mathrm{m})$ and $1370 \mathrm{~cm}^{-1}(7.25 \mu \mathrm{m})$. Based on these comparisons, we conclude the following:

1. The most carbon-rich laboratory analogs which possess clear aliphatic character, but which also have a comparably strong aromatic component, provide the best agreement with the constraints derived from the current interstellar spectrum. Four of the plasma processed materials considered here satisfied all of the criteria derived from the available DISM observations. The results presented here are consistent with a birth site for the diffuse interstellar aliphatic hydrocarbons within the shells of carbon stars such as CRL 618 (Lequeux \& Jourdain deMuizon 1990; Chiar et al. 1998), subject to subsequent competition between hydrogenation and energetic dehydrogenation in the ISM as discussed in Mennella et al. (1999).

2. Infrared spectra of the organic extract from the Murchison meteorite, E. coli bacteria, and ice-processed organic residues reveal spectral features in the 5-10 $\mu \mathrm{m}$ wavelength region which are not within the upper limits of possible features in the spectra of the DISM, and these materials fail to meet the criteria established as an indicator of applicability to the diffuse interstellar dust.

Concerning the meteorite, it remains possible that an unaltered interstellar component lies underneath a more complex suite of materials formed during solar nebula processing or later in the parent body of the meteorite. Such an argument cannot be made for bacteria, however, and we therefore conclude there is no spectral evidence for bacteria in space.

The criteria imposed by the currently available astronomical data are admittedly limited, and additional obser- 
vations are essential. Higher quality interstellar spectra at the longer wavelengths $(5-10 \mu \mathrm{m})$ are needed for Cyg OB2 No. 12 and other lines of sight which sample the DISM. At present, data in the 5-10 $\mu \mathrm{m}$ region is available only for two lines of sight, and Cyg OB2 No. 12 is the only one which samples primarily diffuse ISM material. Additional observations in this wavelength regime are required if we are ever to fully assess the amount of cosmic carbon inside interstellar grains, to reveal the true chemical nature of the organic refractory component of the DISM, and to discriminate further among the various candidate analog materials. The similarity of the aliphatic $\mathrm{CH}$ stretch region of dust from our own Galaxy compared with that of distant galaxies implies that the organic component of the ISM is ubiquitous and similar in nature, but it is essential to measure the $5-10 \mu \mathrm{m}$ spectral region of extragalactic dust as well to investigate the degree of similarity. The aliphatic hydrocarbon signature detected in IRAS $08572+3915$ is the strongest ever recorded (a factor of 4 times stronger than the signature from dust in our own Galaxy; Pendleton 1996a, 1996b), making this galaxy the quintessential target for longer wavelength studies.

We especially wish to thank Cristina Dalle Ore, who spent many hours assembling all the spectral comparison figures and tables presented here. Her help has been invaluable in preparing this manuscript. We are also very grateful to Jason Dworkin and Max Bernstein for their invaluable work, insight, and patience in preparing Figure 17. We are grateful to Dale Cruikshank and Vito Mennella for a careful review of the manuscript. We express thanks to J. Trent, H. Kagawa, and B. Khare for providing us with the IR spectrum of freeze-dried E. coli. We also are especially thankful to all our colleagues who sent us electronic versions of their data, and for the helpful discussions we have had throughout the development of this paper with many of them, especially Vito Mennella, Marla Moore, Reginald Hudson, and Max Bernstein. We specifically thank the following colleagues for assistance in obtaining the data necessary for these comparisons: M. Bernstein, A. Boogert, J. Chiar, L. Colangeli, C. Dudley, W. Duley, D. Furton, J. M. Greenberg, Th. Henning, R. Hudson, G. Lee-Dadswell, A. Li, V. Mennella, M. Moore, M. Palumbo, V. Pirronello, S. Sandford, M. Schnaiter, S. Seahra, A. Scott, G. Strazzulla, A. G. G. M. Tielens, T. Wdowiak, D. C. B. Whittet, A. Witt, G. Wright, S. Wada, and D. Wooden. We thank an anonymous referee for helpful comments and gratefully acknowledge NASA's support for this work with grants from NASA's Exobiology [344-38-12-09 (Y. J. P.); 344-38-12-04 (L. J. A.)], and Long Term Space Astrophysics (399-20-61; L. J. A.) programs.

\section{REFERENCES}

Adamson, A. J., Whittet, D. C. B., \& Duley, W. W. 1990, MNRAS, 243, 400 Allamandola, L. J. 1984, in Galactic and Extragalactic Infrared Spectroscopy, ed. M. Kessler \& P. Phillips (Dordrect: Reidel), 5

Allamandola, L. J., Sandford, S. A., Tielens, A. G. G. M., \& Herbst, T. M. 1992, ApJ, 399, 134

Allamandola, L. J., Sandford, S. A., \& Valero, G. 1988, Icarus, 76, 225

Anders, E., \& Zinner, E. 1993, Meteorics, 28, 490

Bellamy, L. J. 1960, The Infrared Spectra of Complex Molecules (New York: Wiley)

Bernatowicz, T. J., Cowsik, R., Gibbons, P. C., Lodders, K., Fegley, Jr., B., Amari, S., \& Lewis, R. S. 1996, ApJ, 472, 760

Bernstein, M. P., Sandford, S. A., \& Allamandola, L. J. 1994, J. Phys. Chem., 98, 12206

. 1996, ApJ, 472, 127

1997, ApJ, 476, 932

. 2000, ApJ, 542, 894

Bernstein, M. P., Sandford, S. A., Allamandola, L. J., Chang, S., \& Scharberg, M. A. 1995, ApJ, 454, 327

Bowey, J. E., Adamson, A., \& Whittet, D. C. B. 1998, MNRAS, 298, 131

Butchart, I., McFadzean, A. D., Whittet, D. C. B., Geballe, \& Greenberg, J. M. 1986, A\&A, 154, L5

Chiar, J. E., Pendleton, Y. J., Geballe, T., \& Tielens, A. G. G. M. 1998, ApJ, 507,281

Chiar, J. E., \& Tielens, A. G. G. M. 2001, ApJ, 550, L207

Chiar, J. E., Tielens, A. G. G. M., Whittet, D. C. B., Schutte, W. A., Boogert, A. C. A., Lutz, D., van Dishoeck, E. F., \& Bernstein, M. P. 2000, ApJ, 537,749

Cronin, J. R., \& Pizzarello, S. 1990, Geochim. Cosmochim. Acta, 54, 2859

Cronin, J. R., Pizzarello, S., \& Cruikshank, D. P. 1988, in Meteorites and the Early Solar System, ed. J. F. Kerridge \& M. S. Matthews (Tucson: Univ. Arizona Press), 819

Davies, R. E., et al. 1984, Nature, 311, 748

de Graauw, T., et al. 1996, A\&A, 315, L49

Demyk, K., Dartois, E., d'Hendecourt, L., Jourdain de Muizon, M., Heras, A. M., \& Breitfellner, M. 1998, A\&A, 339, 553

DeVries, M. S., Reihs, K., Wendt, H. R., Golden, W. G., Hunziker, H., Fleming, R., Peterson, E., \& Chang, S. 1993, Geochim. Cosmochim. Acta, 57, 933

d'Hendecourt, L. B., \& Allamandola, L. J. 1986, A\&AS, 64, 453

d'Hendecourt, L. B., Allamandola, L. J., Grim, R. J. A., \& Greenberg J. M. 1986, A\&A, 158, 119

Dischler, B., Bubenzer, A., \& Koidl, P. 1983, Solid State Commun., 48, 105 Duley, W. W. 1984, QJRAS, 25, 109

Duley, W. W., Scott, A. D., Seahra, S., Dadswell, G. 1998, ApJ, 503, L183

Ehrenfreund, P., \& Charnley, S. 2000, ARA\&A, 38, 427

Ehrenfreund, P., Robert, F., d'Hendecourt, L., \& Behar, F. 1991, A\&A, 252, 712

Furton, D. G., Laiho, J. W., \& Witt, A. N. 1999, ApJ, 526, 752

Gerakines, P. A., et al. 1999, ApJ, 526, 1062
Gerakines, P. A., Moore, M. H., \& Hudson, R. L. 2000, A\&A, 357, 793

Gillett, F. C., Forrest, W. J., \& Merrill, K. M. 1973, ApJ, 183, 87

Greenberg, J. M., Li, A., Mendoza-Gomez, C. X., Schutte, W. A., Gerakines, P. A., \& De Groot, M. 1995, ApJ, 455, L177

Greenberg, J. M., et al. 2000, ApJ, 531, L71

Greenberg, J. M., \& Munoz Caro, G. M. 2000, in IAU Symp. 197, Astrochemistry: From Molecular Clouds to Planetary Systems, ed. Y. C. Minh \& E. F. van Dishoeck (Dordrecht: Kluwer), 331

Guillois, O., Nenner, I., Papoular, R., \& Reynaud, C. 1996, ApJ, 464, 810

Henning, Th., \& Schnaiter, M. 1999, in Laboratory Astrophysics and Space Research, ed. P. Ehrenfreund, C. Krafft, H. Kochan, \& V. Pirronello (Dordrecht: Kluwer), 249

Hony, S., van Kerckhoven, C., Peeters, E., Tielens, A. G. G. M., Hudgins, D. M., \& Allamandola, L. J. 2001, A\&A, 370, 1030

Hoyle, F., \& Wickramasinghe, N. C. 1991, The Theory of Cosmic Grains (Dordrecht: Kluwer)

. 1993, Our Place in the Cosmos (London: Orion House) 2000, Astronomical Origins of Life: Steps Towards Panspermia (Dordrecht: Kluwer)

Hoyle, F., Wickramasinghe, N. C. Al-Mufti, S., \& Olavesen, A. H. 1982, Ap\&SS, 81, 489

Hudson, R. L., \& Moore, M. 2000, A\&A, 357, 787

Humphreys, R. M. 1978, ApJS, 38, 309

Imanishi, M. 2000, MNRAS, 319, 331

Imanishi, M., \& Dudley, C. C. 2000, ApJ, 545, 701

Innocenti, L., \& Mesland, D. A. M. 1995, Adv. Space Res., 16, 8

Jones, A., Tielens, A. G. G. M., \& Hollenbach, D. 1996, ApJ, 469, 740

Keane, J., et al. 2001, A\&A, 376, 254

Kerridge, J. F., Chang, S., \& Shipp, R. 1987, Geochim. Cosmochim. Acta, 51,2527

Lee, W., \& Wdowiak, T. J. 1993, ApJ, 417, L49

Lequeux, J., \& Jourdain De Muizon, M. 1990, A\&A, 240, L19

Lewis, R. S., Amari, S., \& Anders, E. 1994, Geochim. Cosmochim. Acta, 58, 471

Low, M. J. D., \& Mortera, C. 1983, Carbon, 21, 275

Lutz, D., et al. 1996, A\&A, 315, L269

McKee, C. F. 1989, in Interstellar Dust, ed. L. J. Allamandola \&

A. G. G. M. Tielens (Dordrecht: Kluwer), 431

McMillan, R. S., \& Tapia, S. 1977, ApJ, 212, 714

Mendoza-Gomez, C. X. 1992, Ph.D. thesis, Univ. Leiden

Mennella, V., Brucato, J. R., Colangeli, L., \& Palumbo, P. 1999, ApJ, 524, L71

Mennella, V., Munoz Caro, G. M., Ruiterkamp, R., Schutte, W. A., Greenberg, J. M., Brucato, J. R., \& Colangeli, L. 2001, A\&A, 367, 355

Ming, T., \& Anders, E. 1988, Geochim. Cosmochim. Acta, 52, 1245

Moore, M. H., Hudson, R. L., \& Gerakines, P. A. 2000, Spectrochim. Acta, 57,843

Palumbo, M. E., Pendleton, Y. J., Strazzulla, G. 2000a, ApJ, 542, 890 
Palumbo, M. E., Strazzulla, G., Pendleton, Y. J., Tielens, A. G. G. M. 2000b, ApJ, 534, 801

Papoular, R., et al. 1996, A\&A, 315, 222

Pendleton, Y. J. 1995, Planet. Space Sci., 43, 1359 1996a, in The Cosmic Dust Connection, ed. J. M. Greenberg (Dordrecht: Kluwer), 71

1996b, in New Extragalactic Perspectives in the New South Africa, ed. D. Block \& J. M. Greenberg, (Dordrecht:Kluwer), 135

1997, Origins Life Evol. Biosphere, 27, 53

Pendleton, Y. J., Sandford, S. A., Allamandola, L. J., Tielens, A. G. G. M., \& Sellgren, K. 1994, ApJ, 437, 683

Pendleton, Y. J., Tielens, A. G. G. M., Tokunaga, A. T., \& Bernstein, M. P. 1999, ApJ, 513, 294

Persi, P., \& Ferrari-Toniolo, M. 1982, A\&A, 111, L7

Pirronello, V., Liu, C., Roser, J. E., \& Vidali, G. 1999, A\&A, 344, 681

Rieke, G. H. 1974, ApJ, 193, L81

Roche, P. F., \& Aitken, D. K. 1984, MNRAS, 208, 481 1985, MNRAS, 215, 425

Sakata, A., \& Wada, S. 1989, in IAU Symp. 135, Interstellar Dust, ed. L. J. Allamandola \& A. G. G. M. Tielens (Dordrecht: Kluwer), 191

Sakata, A., Wada, S., Okutsu, Y., Shintani, H., \& Nakada, Y. 1983, Nature, 301,493

Sakata, A., Wada, S., Onaka, T., \& Tokunaga, A. T. 1987, ApJ, 320, L63

Sandford, S. A., Allamandola, L. J., Tielens, A. G. G. M., Sellgren, K., Tapia, M., \& Pendleton, Y. J. 1991, ApJ, 371, 607

Sandford, S. A., Pendleton, Y. J., \& Allamandola, L. J. 1995, ApJ, 440, 697

Schnaiter, M., et al. 1998, ApJ, 498, 486 1999, ApJ, 519, 687

Schutte, W. A., et al. 1998, A\&A, 337, 261
Scott, A. D. 1997, Ph.D. thesis, Univ. Waterloo

Scott, A., \& Duley, W. W. 1996, ApJ, 472, L123

Scott, A., Duley, W. W., \& Pinho, G. P. 1997, ApJ, 489, L193

Silverstein, R. M., \& Bassler, G. C. 1967, Spectrometric Identification of Organic Compounds (New York: Wiley)

Sloan, G. C., Bregman, J. D., Geballe, T. R., Allamandola, L. J., Woodward, Charles E. 1997, ApJ, 474, 735

Strazzulla, G., Baratta, G. A., \& Magazzu, A. 1991, in Solid State Astrophysics, ed. E. Busoletti \& G. Strazzulla (Amsterdam: North Holland), 403

Tielens, A. G. G. M. 1998, ApJ, 499, 267

Tielens, A. G. G. M., Wooden, D. H., Allamandola, L. J., Bregman, J., \& Witteborn, F. C. 1996, ApJ, 461, 210

Torres-Dodgen, A. V., Carroll, M., \& Tapia, M. 1991, MNRAS, 249, 1

Wexler, A. S. 1967, Appl. Spectrosc. Rev., 1, 29

Whittet, D. C. B. 1984, Observatory, 104, 159

. 1997, Origins Life Evol. Biosphere, 27, 101

Whittet, D. C. B., Pendleton, Y. J., Gibb, E. L., Boogert, A. C. A., Chiar, J. E., \& Nummelin, A. 2001, ApJ, 550, 793

Whittet, D. C. B., \& Tielens, A. G. G. M. 1997, in From Stardust to Planetesimals, ed. Y. J. Pendleton \& A. G. G. M. Tielens (San Francisco: ASP), 161

Whittet, D. C. B., et al. 1997, ApJ, 490, 729

Wickramasinghe, D. T., \& Allen, D. A. 1980, Nature, 287, 518

Willner, S. P., Russell, R. W., Puetter, R. C., Soifer, B. T., \& Harvey, P. M. 1979, ApJ, 229, L65

Wright, G., Geballe, T., Bridger, A., \& Pendleton, Y. P. 1996, in New Extragalactic Perspectives in the New South Africa, ed. D. Block \& J. M. Greenberg (Dordrecht: Kluwer), 143 